\title{
Transmitter Linearization by Beamforming
}

\author{
ChuanKang Liang, Member, IEEE, and Behzad Razavi, Fellow, IEEE
}

\begin{abstract}
Millimeter-wave transmitters designed for dense signal constellations must deal with severe linearity-efficiency trade-offs. This paper proposes a method of blending beamforming and linearization that reduces the number of power amplifiers and avoids the loss of on-chip transformers. Two constant-envelope beams are combined in space to deliver a variable-envelope signal, relaxing the linearity of transmitters. A dual-transmitter prototype fabricated in 65-nm CMOS technology and designed for the $60-\mathrm{GHz}$ band produces a 16QAM output of $+9.7 \mathrm{dBm}$ with $11 \%$ efficiency.
\end{abstract}

Index Terms-Beamforming, injection-locked power amplifier, millimeter-wave circuits, $\mathbf{m m}$-wave phased-array transmitter, outphasing technique, phase shifter, transmitter linearization.

\section{INTRODUCTION}

D ENSE signal constellations such as 16QAM or 64QAM are often used to alleviate the trade-off between the bandwidth and the data rate. Such constellations, however, impose other constraints on transceiver design: transmitter (TX) and receiver (RX) nonlinearity, carrier phase noise, and baseband data converter resolution. The issue of TX nonlinearity is tackled by means of either back-off from compression or linearization techniques, both of which face difficulties at millimeter-wave (mm-wave) frequencies.

This paper describes a TX architecture that combines beamforming and linearization, allowing the use of nonlinear (and hence more efficient) power amplifiers (PAs) with variable-envelope signals. Designed for the 60-GHz band in 65-nm CMOS technology, an experimental prototype includes two transmitters, a phase-locked loop (PLL), and analog baseband phase shifters. Using nonlinear, self-oscillating PAs, the dual-TX system produces a 16QAM signal that, upon beamforming, yields an error vector magnitude (EVM) of $-21.6 \mathrm{dBm}$.

Section II of the paper provides the background for this work and Section III introduces the architecture. Section IV describes the design of the building blocks and Section $\mathrm{V}$ presents the experimental results.

\section{BACKGROUND}

A common approach to transmitting amplitude- and phase-modulated signals, especially at very high frequen-

Manuscript received December 19, 2009; revised March 09, 2011; accepted April 03, 2011. Date of publication June 13, 2011; date of current version August 24, 2011. This paper was approved by Associate Editor Ranjit Gharpurey.

C. Liang was with the Electrical Engineering Department, University of California at Los Angeles, and is now with Mediatek USA, San Jose, CA 95134 USA.

B. Razavi is with the Electrical Engineering Department, University of California at Los Angeles, Los Angeles, CA 90095-1594 USA (e-mail: razavi@ee. ucla.edu).

Color versions of one or more of the figures in this paper are available online at http://ieeexplore.ieee.org.

Digital Object Identifier 10.1109/JSSC.2011.2148530

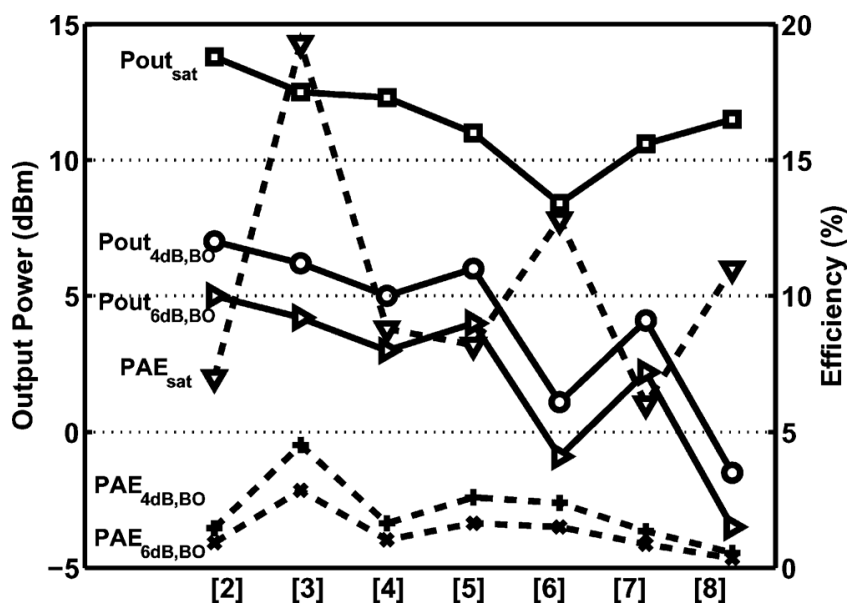

Fig. 1. Performance of mm-wave CMOS PAs before and after back-off.

cies, is to back-off from the output 1-dB compression point $\left(P_{1 \mathrm{~dB}}\right)$ of the transmitter by a sufficient margin. For example, a 16QAM waveform exhibits a peak-to-average ratio (PAR) of about $2.5 \mathrm{~dB}$ [1], and typical baseband pulse-shaping raises the PAR by another 2 to $5 \mathrm{~dB}[1],{ }^{1}$ requiring considerable back-off. (A detailed analysis is presented in Appendix I.)

The principal drawback of back-off is the drastic fall in both the output power, $P_{\text {out }}$, and the PA efficiency, e.g., the drain efficiency, $\eta$. Fig. 1 plots the saturated $P_{\text {out }}$ and $\eta$ for a number of published mm-wave CMOS PAs along with the values at 4-dB and 6-dB back-off from $P_{1 \mathrm{~dB}}$. It is observed that, even for 4-dB back-off, $P_{\text {out }}$ and $\eta$ remain below $7 \mathrm{dBm}$ and $4.6 \%$, respectively.

An alternative approach is to apply explicit linearization techniques to the TX, specifically, to the PA. Among the multitude of linearization techniques, two do not require any linearity ${ }^{2}$ in the PA and have emerged as viable solutions for integrated circuits: polar modulation [also known as "envelope elimination and restoration" (EER)] [9] and outphasing ${ }^{3}$ [also known as "linear amplification with nonlinear components" (LINC)] [10], [11]. Illustrated in Fig. 2(a), EER decomposes the signal into a phase-modulated (PM) component and an envelope, amplifies them, and impresses the envelope on the phase signal at the output. EER must deal with a number of issues, especially at high data rates: 1) matching the delay between the phase and amplitude paths is difficult because the circuits in these two paths have very different time constants; 2) the bandwidths of the envelope and phase paths must be quite larger than that of the original signal [12], [13]; and 3) as conceptually shown in Fig. 2(b), the envelope modulation path at the output node sus-

\footnotetext{
${ }^{1}$ For example, a root-raised-cosine filter with a roll-off factor of 0.4 raises PAR to $3.5 \mathrm{~dB}[1]$.

${ }^{2}$ If $\mathrm{AM} / \mathrm{PM}$ conversion is negligible.

${ }^{3}$ The rate at which the efficiency of outphasing PAs degardes with back-off is similar to that of class-A stages.
} 


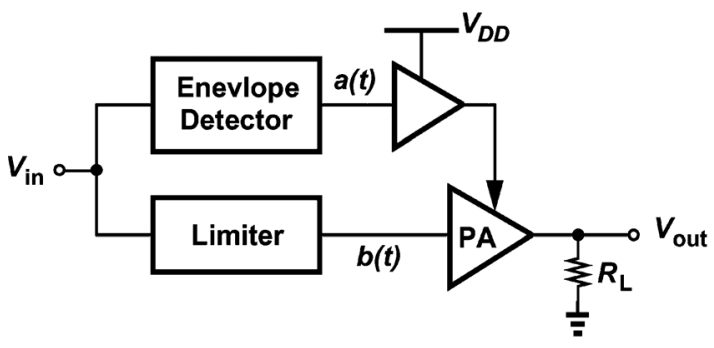

(a)

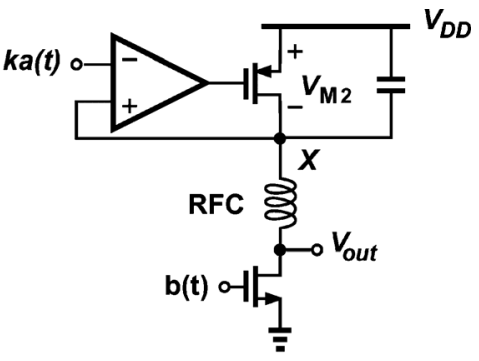

(b)

Fig. 2. (a) EER architecture and (b) representative EER output stage.

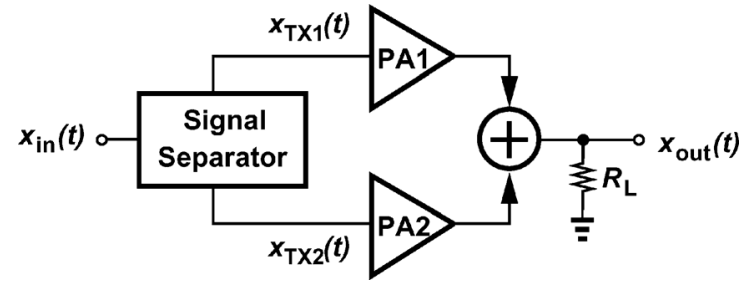

Fig. 3. Generic outphasing architecture.

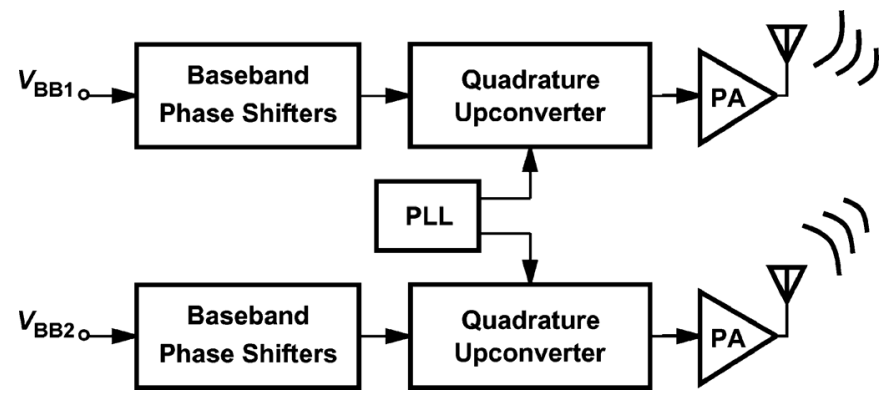

Fig. 4. Proposed dual-transmitter outphasing architecture.

tains a certain amount of voltage, $V_{M 2}$, limiting the headroom and degrading the efficiency at low supply voltages.

Fig. 3 depicts the generic outphasing technique. The RF signal, $x_{\text {in }}(t)=a(t) \cos \left[\omega_{c}+\phi(t)\right]$, is represented by two constant-envelope components:

$$
\begin{aligned}
& x_{\mathrm{TX} 1}(t)=\frac{A_{\max }}{2} \cos \left[\omega_{c} t+\phi(t)+\psi(t)\right] \\
& x_{\mathrm{TX} 2}(t)=\frac{A_{\max }}{2} \cos \left[\omega_{c} t+\phi(t)-\psi(t)\right]
\end{aligned}
$$

where $A_{\max }=\max |a(t)|$ and $\psi(t)=\cos ^{-1}\left[a(t) / A_{\max }\right]$. We call $\psi(t)$ the "outphasing angle." These two components are amplified and summed at the output so as to yield the composite signal. In contrast to polar modulation, outphasing employs identical signal paths, more easily achieving small phase mismatches, and requires no supply modulation at the output, avoiding the headroom and efficiency degradation associated therewith.

The outphasing arrangement of Fig. 3 entails its own issues. 1) Direct decomposition of RF signal to $x_{\mathrm{TX} 1}$ and $x_{\mathrm{TX} 2}$ proves difficult, particularly at mm-wave frequencies, making separation in the transmitter baseband more attractive. 2) Coupling between the PA outputs through the output combining network distorts $x_{\mathrm{TX} 1}(t)$ and $x_{\mathrm{TX} 2}(t)$ if the PAs do not have a zero output impedance. As originally derived by Chireix [10], this coupling,

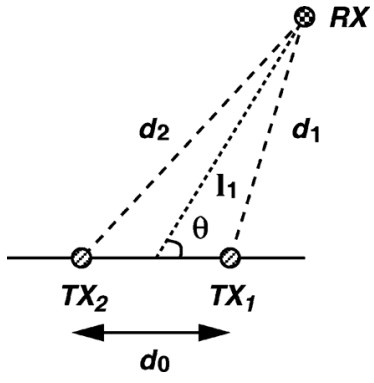

Fig. 5. A phased array with two transmitters.

in the absence of envelope modulation, equivalently introduces a capacitance at the output of $\mathrm{PA}_{1}$, and an inductance at the output of $\mathrm{PA}_{2}$ (or the other way around). With envelope modulation, these parasitics act as time-varying reactances, thereby distorting the phases of $x_{\mathrm{TX} 1}$ and $x_{\mathrm{TX} 2}$.

It has also been recognized that, since a passive combining network cannot provide both a zero loss and a zero coupling between its input ports [14], outphasing suffers from a trade-off between the output combiner loss and signal distortion. ${ }^{4}$ In addition, on-chip combiners, e.g., transformers, suffer from an intrinsic loss of 1 or $2 \mathrm{~dB}$ even if isolation is not a concern.

\section{Proposed Architecture}

The loss of on-chip transformers in an outphasing system can be avoided if the outputs of the transmitters are combined in space rather than on the chip or on the board. Based on this concept, the architecture of Fig. 4 is proposed. Here, two (or more) transmitters that would be necessary in a generic beamforming system are reconfigured so as to produce constant-envelope outphasing signals, and the outputs are combined at a certain angle in space so as to reconstruct the original amplitude- and phase-modulated signal. While the concept is applicable to any carrier frequency, it is implemented in this work in the unlicensed $60-\mathrm{GHz}$ band because the high path loss at these frequencies makes beamforming particularly desirable.

In this section, we analyze the proposed architecture, consider systems employing more than two transmitters, and formulate the effect of nonidealities.

\section{A. Basic Operation}

Consider the arrangement shown in Fig. 5, where the transmitting antennas have a spacing of $d_{0}$ and the receiving antenna

${ }^{4}$ For example, a Wilkinson combiner must employ a bridge resistor between its inputs to provide isolation [15], wasting power when the two inputs are not identical. 
is located at a distance of $l_{1}$ and angle of $\theta$ with respect to the midpoint between $\mathrm{TX}_{1}$ and $\mathrm{TX}_{2}$. We have

$$
\begin{aligned}
d_{1} & =\sqrt{l_{1}^{2}+\left(\frac{d_{0}}{2}\right)^{2}-l_{1} d_{0} \cos \theta} \\
& \approx l_{1} \sqrt{1-\frac{d_{0} \cos \theta}{l_{1}}} \\
& \approx l_{1}-\frac{d_{0} \cos \theta}{2}
\end{aligned}
$$

if $l_{1} \gg d_{0}$. Similarly,

$$
d_{2} \approx l_{1}+\frac{d_{0} \cos \theta}{2} .
$$

We assume the waveforms expressed by (1) and (2) and note that $x_{\mathrm{TX} 1}(t)$ arrives at the $\mathrm{RX}$ as $x_{\mathrm{TX} 1}\left(t-d_{1} / v_{c}\right)$, where $v_{c}$ denotes the speed of light, and $x_{\mathrm{TX} 2}(t)$ as $x_{\mathrm{TX} 2}\left(t-d_{2} / v_{c}\right)$. Addition of the two received waveforms yields equation (5), shown at the bottom of the page, where $\tau=t+l_{1} / v_{c}$ and $\alpha$ represents the propagation loss. As explained in Section IV-A, $\phi\left[\tau+d_{0} \cos \theta /\left(2 v_{c}\right)\right] \approx \phi(\tau)$ and $\psi\left[\tau+d_{0} \cos \theta /\left(2 v_{c}\right)\right] \approx \psi(\tau)$ in typical cases and hence

$$
\begin{aligned}
x_{\mathrm{RX}}(\tau) \approx & \frac{\alpha A_{\max }}{2} \cos \left[\omega_{c} \tau+\phi(\tau)+\psi(\tau)+\omega_{c} \frac{d_{0} \cos \theta}{2 v_{c}}\right] \\
& +\frac{\alpha A_{\max }}{2} \cos \left[\omega_{c} \tau+\phi(\tau)-\psi(\tau)-\omega_{c} \frac{d_{0} \cos \theta}{2 v_{c}}\right]
\end{aligned}
$$$$
=\alpha A_{\max } \cos \left[\psi(\tau)+\omega_{c} \frac{d_{0} \cos \theta}{2 v_{c}}\right] \cos \left[\omega_{c} \tau+\phi(\tau)\right]
$$

indicating that $x_{\mathrm{RX}}(\tau)$ reduces to the desired waveform, $\alpha a(\tau) \cos \left[\omega_{c} \tau+\phi(\tau)\right]$, if $\theta=90^{\circ}$. For reception at other spatial angles, $\mathrm{TX}_{1}$ or $\mathrm{TX}_{2}$ must introduce an additional phase shift in its output signal. ${ }^{5}$

An important advantage of the proposed architecture is that it halves the number of PAs necessary for outphasing. That is, a conventional beamforming system employing two independent outphasing transmitters would require four PAs and entail more complex layout and routing issues, especially at mm-wave frequencies. ${ }^{6}$

\section{B. Security Feature}

The additional phase, $\omega_{c} d_{0} \cos \theta / 2 v_{c}$, in the argument of the first cosine in (7) corrupts the received signal, revealing that, if the receiver is located at a spatial angle at which this term

${ }^{5}$ Calculations and simulations indicate that the effect of coupling between the antennas negligibly affects the overall signal quality if the coupling factor is less than $-20 \mathrm{~dB}$.

${ }^{6}$ Unlike conventional beamforming systems, this approach introduces interference in other directions.

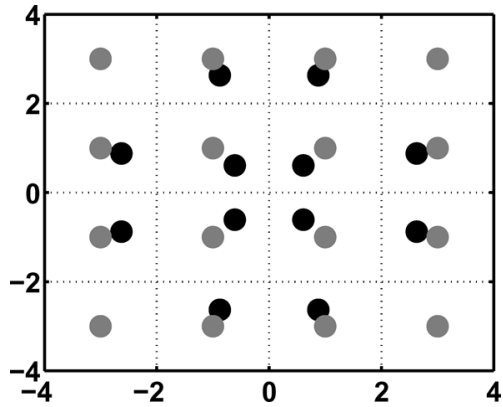

Fig. 6. Simulated received 16QAM constellation at $\theta=90^{\circ}$ (gray dots) and $\theta=85^{\circ}$ (black dots)

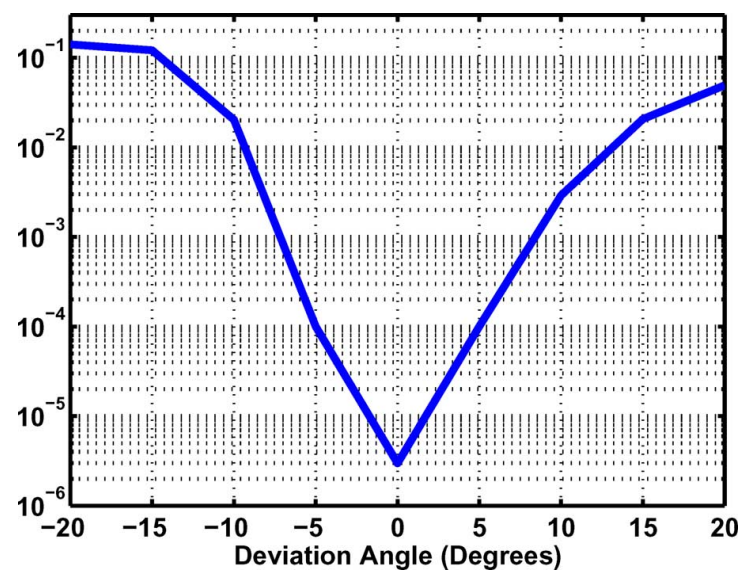

Fig. 7. Simulated BER at different deviation angles with $\mathrm{SNR}=20 \mathrm{~dB}$ for a 16QAM signal.

does not vanish, then the composite received signal is corrupted. In other words, receivers located outside a certain solid angle cannot easily decipher the information that is transmitted by $\mathrm{TX}_{1}$ and $\mathrm{TX}_{2}$.

For small deviations from the ideal receiving angle, $\omega_{c} d_{0} \cos \theta / 2 v_{c}$ can be assumed much less than 1 radian, allowing (7) to be written as

$$
\begin{aligned}
& x_{\mathrm{RX}}(\tau) \\
& \approx \alpha A_{\max }\left\{\cos [\psi(\tau)]-\omega_{c} \frac{d_{0} \cos \theta}{2 v_{c}} \sin [\psi(\tau)]\right\} \cos \left[\omega_{c} \tau+\phi(\tau)\right] \\
& \approx \alpha\left[a(\tau)-\omega_{c} \frac{d_{0} \cos \theta}{2 v_{c}} A_{\max } \sqrt{1-\frac{a^{2}(t)}{A_{\max }^{2}}}\right] \cos \left[\omega_{c} \tau+\phi(\tau)\right] .
\end{aligned}
$$

Thus, the envelope is corrupted by its harmonics. As an example, Fig. 6 shows a simulated 16QAM constellation at $5^{\circ}$ away from the ideal receiving angle, indicating that the EVM rises to $-13.2 \mathrm{~dB}$ even in the absence of noise. Based on (7), Fig. 7 plots the bit error rate (BER) as a function of the deviation angle (with respect to $\theta=90^{\circ}$ ) for a 1-GHz 16QAM

$$
\begin{aligned}
x_{\mathrm{RX}}(\tau)= & \frac{\alpha A_{\max }}{2} \cos \left[\omega_{c} \tau+\phi\left(\tau+\frac{d_{0} \cos \theta}{2 v_{c}}\right)+\psi\left(\tau+\frac{d_{0} \cos \theta}{2 v_{c}}\right)+\omega_{c} \frac{d_{0} \cos \theta}{2 v_{c}}\right] \\
& +\frac{\alpha A_{\max }}{2} \cos \left[\omega_{c} \tau+\phi\left(\tau-\frac{d_{0} \cos \theta}{2 v_{c}}\right)-\psi\left(\tau-\frac{d_{0} \cos \theta}{2 v_{c}}\right)-\omega_{c} \frac{d_{0} \cos \theta}{2 v_{c}}\right]
\end{aligned}
$$




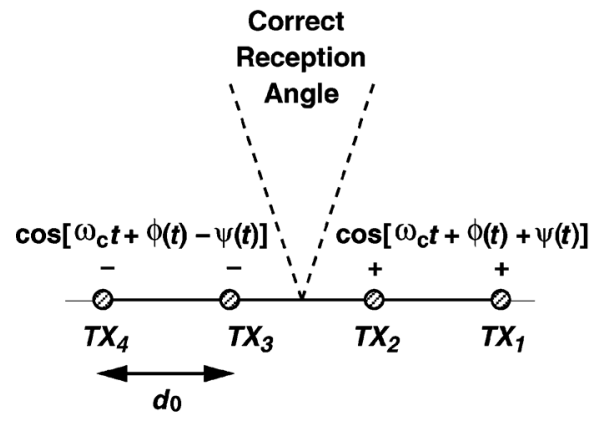

(a)

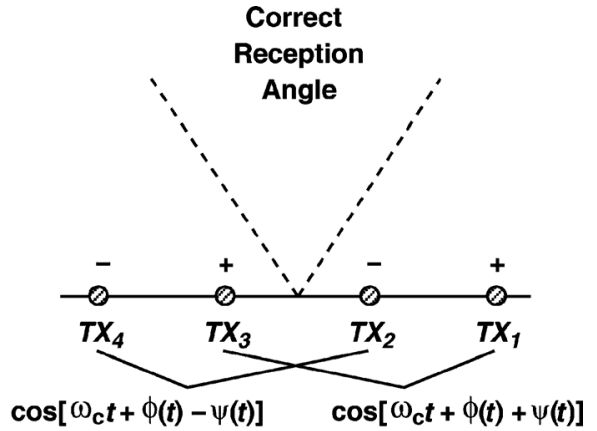

(b)

Fig. 8. Four-transmitter phased array for (a) odd-symmetric assignment of $\psi(t)$, and (b) alternating assignment of $\psi(t)$.

signal with baseband pulse shaping, an SNR of $20 \mathrm{~dB}$, a carrier frequency of $60 \mathrm{GHz}$, and $d_{0}=\lambda / 2$. We observe that for angles greater than about $\pm 10^{\circ}$, little intelligible information is received. Note that the received spectrum also broadens as the deviation angle increases. Absent in conventional beamforming systems, this property can be utilized for secure communication in a narrow solid angle. ${ }^{7}$

\section{Larger Number of Transmitters}

The architecture of Fig. 5 can be extended to a larger number of transmitters. Shown in Fig. 8 are two scenarios for the case of four antennas. In Fig. 8(a), $\mathrm{TX}_{1}$ and $\mathrm{TX}_{2}$ produce one component of the outphasing signals, e.g., according to (1), and $\mathrm{TX}_{3}$ and $\mathrm{TX}_{4}$, the other, e.g., according to (2). The received signal at an angle of $\theta$ is thus given by

$$
\begin{aligned}
& x_{\mathrm{RX}}(\tau)=2 \alpha A_{\max } \cos \left[\psi(\tau)+\omega_{c} \frac{d_{0} \cos \theta}{v_{c}}\right] \\
& \times \cos \left[\omega_{c} \tau+\phi(\tau)\right] \cos \left(\omega_{c} \frac{d_{0} \cos \theta}{2 v_{c}}\right) .
\end{aligned}
$$

Since the corruption term is doubled, the solid angle for correct reception is approximately equal to half that found in the previous section for two antennas. On the other hand, if, as depicted in Fig. 8(b), the polarity of the outphasing angle, $\psi(\tau)$, alternates from one TX to the next, then

$$
\begin{aligned}
x_{\mathrm{RX}}(\tau)=2 \alpha A_{\max } \cos \left[\psi(\tau)+\omega_{c} \frac{d_{0} \cos \theta}{2 v_{c}}\right] \\
\times \cos \left[\omega_{c} \tau+\phi(\tau)\right] \cos \left(\omega_{c} \frac{d_{0} \cos \theta}{v_{c}}\right) .
\end{aligned}
$$

In this case, a wider solid angle is provided for reception. ${ }^{8}$

\section{Effect of Nonidealities}

In the architecture of Fig. 4, amplitude and phase mismatches between the two transmitters and within each transmitter corrupt the overall transmitted signal. In this section, we formulate the effect of these mismatches.

\footnotetext{
${ }^{7}$ In contrast to the antenna modulation technique in [16], our approach does not need to reconfigure the antennas by switched reflectors.

${ }^{8}$ As one of the reviewers has pointed out, this result can also be derived by noting that the spacing between the $\pm \psi$ antennas is halved in Fig. 8(b), thus doubling the beamwidth.
}

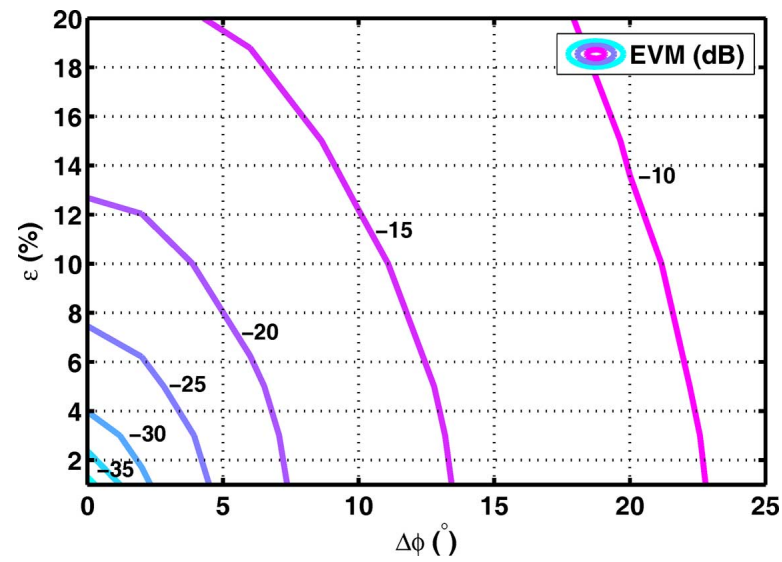

Fig. 9. Simulated EVM contours with amplitude and phase mismatches.

Let us write the signal received from the two transmitters in Fig. 5 at $\theta=90^{\circ}$ as

$$
\begin{gathered}
x_{\mathrm{RX}}(\tau)=\alpha \frac{A_{\max }}{2}\left(1+\frac{\epsilon}{2}\right) \cos \left[\omega_{c} \tau+\phi(\tau)+\psi(\tau)+\frac{\Delta \phi}{2}\right] \\
+\alpha \frac{A_{\max }}{2}\left(1-\frac{\epsilon}{2}\right) \cos \left[\omega_{c} \tau+\phi(\tau)-\psi(\tau)-\frac{\Delta \phi}{2}\right]
\end{gathered}
$$

where $\epsilon$ and $\Delta \phi$ denote amplitude and phase mismatches, respectively. It is important to note that these mismatches model both those between the transmitters and those between the propagation paths from $\mathrm{TX}_{1}$ and $\mathrm{TX}_{2}$ to the receiver. Expanding the cosines and assuming $\epsilon \ll 1$ and $\Delta \phi \ll 1 \mathrm{rad}$, we have

$$
\begin{gathered}
x_{\mathrm{RX}}(\tau)=\alpha\left[a(\tau)-\frac{\Delta \phi}{2} \sqrt{A_{\max }^{2}-a^{2}(\tau)}\right] \cos \left[\omega_{c} \tau+\phi(\tau)\right] \\
-\alpha \frac{\epsilon}{2}\left[a(\tau) \frac{\Delta \phi}{2}+\sqrt{A_{\max }^{2}-a^{2}(\tau)}\right] \sin \left[\omega_{c} \tau+\phi(\tau)\right] .
\end{gathered}
$$

Thus, amplitude mismatch introduces an additive quadrature component with a distorted envelope (the second term), and phase mismatch creates envelope distortion in the main signal (the first term). The phase mismatch, of course, is readily removed as the system adjusts the direction of the beam toward the receiver.

Fig. 9 plots the constant-EVM contours for a 16QAM signal with amplitude and phase mismatches in the transmitter paths. We observe that an EVM of lower than $-20 \mathrm{~dB}$ can be achieved if, e.g., $\epsilon=10 \%$ and $\Delta \phi=6^{\circ}$. If these values are difficult to achieve at mm-wave frequencies, each TX can incorporate both amplitude and phase control so that, in the initial handshaking 


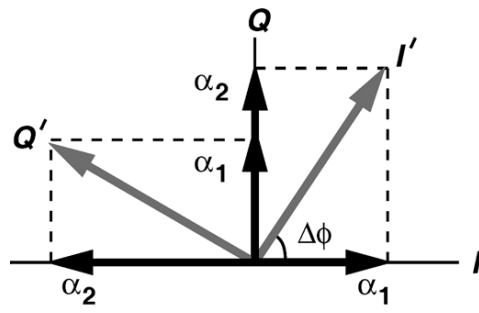

(a)

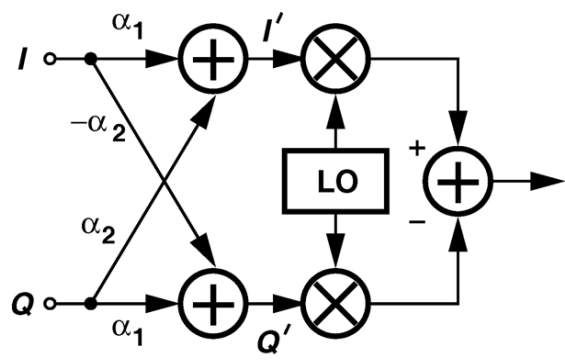

(b)

Fig. 10. (a) The concept and (b) realization of $I / Q$ interpolation in baseband.

between the RX and two transmitters, both quantities are adjusted for the highest received SNR.

The mismatches within each TX manifest themselves as in conventional transmitters, i.e., by stretching the signal constellation in one direction and compressing it in another direction [17]. This effect can be formulated as follows.

$$
\begin{aligned}
x_{\mathrm{RX}}(\tau)= & {[1+(1+\epsilon) \cos \Delta \phi] a(\tau) \cos \left[\omega_{\mathrm{LO}} \tau+\phi(\tau)\right] } \\
& +[-1+(1+\epsilon) \cos \Delta \phi] a(\tau) \cos \left[\omega_{\mathrm{LO}} \tau-\phi(\tau)\right] \\
& -(1+\epsilon) \sin \Delta \phi a(\tau) \sin \left[\omega_{\mathrm{LO}} \tau+\phi(\tau)\right] \\
& -(1+\epsilon) \sin \Delta \phi a(\tau) \sin \left[\omega_{\mathrm{LO}} \tau-\phi(\tau)\right]
\end{aligned}
$$

where $\epsilon$ and $\Delta \phi$ respectively denote the gain and phase mismatches between the I and Q paths (including those of the LO).

\section{BUILDING BLOCKS}

This section presents the design of the building blocks of the two-transmitter prototype in 65-nm CMOS technology. These blocks include the baseband phase shift circuit, the quadrature upconversion mixers, the PLL, and the power amplifier.

\section{A. Phase Shift Circuit}

An integral part of beamforming systems is the phase shift operation required to compensate for unequal propagation times of the beams. The phase shift can be inserted in the RF or LO paths [18]-[20]. At mm-wave frequencies, however, the trade-off between the phase shift and the loss of the passive networks (or between the phase shift and the power consumption of active networks) limits the utility of theses techniques. This issue becomes particularly serious in CMOS technology due to the MOSFETs' poor drive capability. It is therefore desirable to realize the phase shift in the baseband [21], [22].

Suppose two transmitted signals in a conventional beamforming system experience unequal propagation delays upon reception. For example,

$$
\begin{aligned}
x_{1}(t) & =a(t) \cos \left[\omega_{c} t+\phi(t)\right] \\
x_{2}(t) & =a(t-\Delta T) \cos \left[\omega_{c}(t-\Delta T)+\phi(t-\Delta T)\right] \\
& =a(t-\Delta T) \cos \left[\omega_{c} t+\phi(t-\Delta T)-\omega_{c} \Delta T\right] .
\end{aligned}
$$

The key observation here is that, for $x_{1}(t)$ to be aligned with $x_{2}(t)$, it must undergo three changes: $a(t)$ must be shifted by $\Delta T ; \phi(t)$ must be shifted by $\Delta T$; and a constant phase equal to $-\omega_{c} \Delta T$ must be added to the carrier. If $a(t)$ and $\phi(t)$ vary slowly enough that $a(t-\Delta T) \approx a(t)$ and $\phi(t-\Delta T) \approx \phi(t)$, then the constant phase suffices to align $x_{1}$ with $x_{2}$. We therefore say the phase shift approximates the delay for a narrowband signal.

On the other hand, if $a(t)$ and $\phi(t)$ vary significantly in $\Delta T$ seconds, then $a(t)$ and $\phi(t)$ or, equivalently, the quadrature components $a(t) \cos [\phi(t)]$ and $a(t) \sin [\phi(t)]$, must be shifted by $\Delta T$ in addition to the constant phase of $-\omega_{c} \Delta T$ that must be impressed on the carrier. The concept of "true time delay" [23] is in fact the same as this shift of $t$ by $\Delta T$ in $a(t) \cos \left[\omega_{c} t+\phi(t)\right]$.

Let us now focus on the realization of the constant phase $-\omega_{c} \Delta T$ in the baseband. If the quadrature baseband components are transformed to $a(t) \cos \left[\phi(t)-\omega_{c} \Delta T\right]$ and $a(t) \sin \left[\phi(t)-\omega_{c} \Delta T\right]$, then after upconversion, we obtain $a(t) \cos \left[\omega_{c} t+\phi(t)-\omega_{c} \Delta T\right]$. A natural implementation emerges if we expand the desired baseband components as $a(t) \cos \phi(t) \cos \left(\omega_{c} \Delta T\right)+a(t) \sin \phi(t) \sin \left(\omega_{c} \Delta T\right)$ and $a(t) \sin \phi(t) \cos \left(\omega_{c} \Delta T\right)-a(t) \cos \phi(t) \sin \left(\omega_{c} \Delta T\right)$, respectively, observing that the original baseband waveforms can be simply scaled and summed, i.e., interpolated.

The approach adopted in this paper interpolates between the baseband quadrature components so as to generate signals of the form $\cos \left[\phi(t)+\psi(t)-\omega_{c} \Delta T\right]$ and $\sin \left[\phi(t)+\psi(t)-\omega_{c} \Delta T\right]$. As illustrated in Fig. 10(a), weighted sums of the baseband I and Q signals produce $\mathrm{I}^{\prime}$ and $\mathrm{Q}^{\prime}$ :

$$
\begin{gathered}
I^{\prime}=\alpha_{1} I+\alpha_{2} Q=\sqrt{\alpha_{1}^{2}+\alpha_{2}^{2}} \cos \left[\phi(t)+\psi(t)-\tan ^{-1} \frac{\alpha_{2}}{\alpha_{1}}\right] \\
Q^{\prime}=-\alpha_{2} I+\alpha_{1} Q=\sqrt{\alpha_{1}^{2}+\alpha_{2}^{2}} \sin \left[\phi(t)+\psi(t)-\tan ^{-1} \frac{\alpha_{2}}{\alpha_{1}}\right] .
\end{gathered}
$$

Thus, $\tan ^{-1}\left(\alpha_{2} / \alpha_{1}\right)$ can be chosen equal to $\omega_{c} \Delta T$. Fig. 10(b) shows the realization of the concept.

We should also remark that baseband interpolation does not preclude beamforming of wideband signals. We can simply shift each baseband component by $\Delta T$ seconds (a few nanoseconds for a maximum path length difference of $1 \mathrm{~m}$ ) in the analog or digital domain, generating $\cos [\phi(t-\Delta T)+\psi(t-\Delta T)]$ and $\sin [\phi(t-\Delta T)+\psi(t-\Delta T)]$ before interpolation. For a maximum path length difference of a few tens of millimeters (a common case for a large phased array at $60 \mathrm{GHz}$ ), $\Delta T$ is on the order of 50-100 picoseconds, suggesting that it is not even necessary for data rates up to a few gigabits per second. 


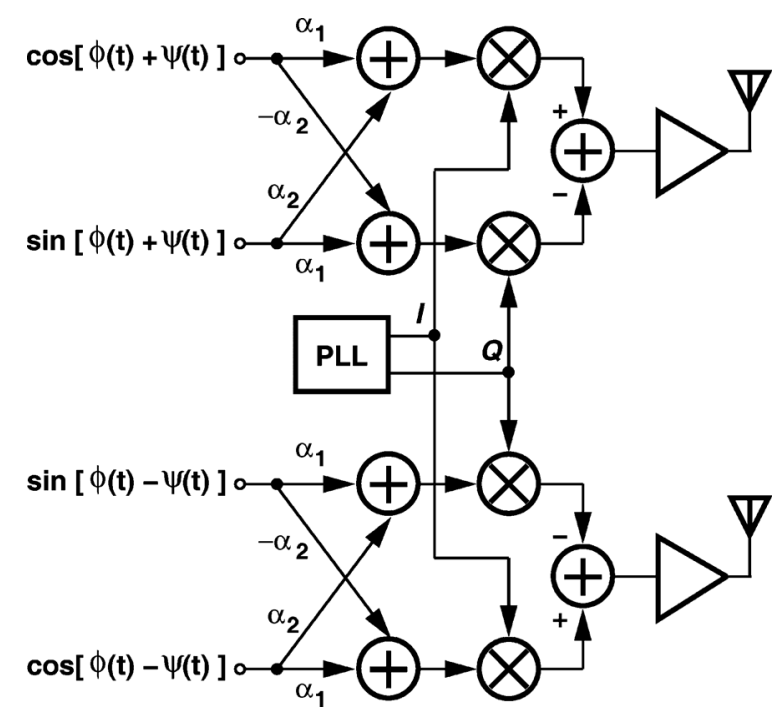

Fig. 11. Proposed dual-TX prototype.

Fig. 11 shows the functional diagram of the dual-TX prototype, depicting the analog baseband signals applied to each transmitter. A drawback of outphasing (with on-chip or off-chip combining) is that it doubles the number of the baseband digital-to-analog converters (DACs) and filters with respect to conventional transmitters. Fortunately, simple extrapolation from [24] suggests that DACs with a resolution of 6 to 8 bits and a clock rate of $1 \mathrm{GHz}$ (to allow a data rate of $4 \mathrm{~Gb} / \mathrm{s}$ with 16QAM) would consume less than $10 \mathrm{~mW} .{ }^{9}$ The filters following the DACs can be omitted [25] but the zero-order-hold output waveform of the DACs occupies a very wide spectrum, thus producing an RF signal that heavily spills onto adjacent channels. A simple second- or third-order filter can minimize this effect.

Fig. 12 shows the details of the interpolation network and its interface with the upconversion mixers. The overall circuit for each TX consists of four differential pairs (i.e., two doublebalanced mixers) driven by $L O_{I}, \overline{\mathrm{LO}_{\mathrm{I}}}, L O_{Q}$, and $\overline{\mathrm{LO}_{\mathrm{Q}}}$, but only one slice is shown for simplicity. The baseband section consists of binary-weighted transistors that convert $V_{B B, I}$ and $V_{B B, Q}$ to current and sum the results at node $\mathrm{X}$. The interpolation weighting factors, $\alpha_{1}$ and $\alpha_{2}$, are set by enabling or disabling the binary-weighted transistors. In this prototype, $m=4$, providing an angular resolution of about $12^{\circ}$. Fig. 12(b) conceptually shows the configuration of the baseband devices for a few phases, revealing that the mixer bias current remains constant. ${ }^{10}$ Note that interpolation incurs no power penalty: the total current drawn from $\mathrm{X}$ is the same as that necessary in a standard upconversion mixer.

Even though sensing constant-envelope inputs, the interpolation network must still be sufficiently linear. Specifically, third-order nonlinearity generates two terms given by $k\left(A_{\max } / 2\right) \cos [3 \phi(t)+3 \psi(t)]$ and $k\left(A_{\max } / 2\right) \cos [3 \phi(t)-$

\footnotetext{
${ }^{9}$ It is assumed that on-chip DACs need not drive a $50-\Omega$ load.

${ }^{10}$ Due to the summation of the baseband quadrature inputs, the signal current does vary to some extent, yielding a $3-\mathrm{dB}$ variation of the mixer output. However, owing to the nonlinear behavior of the PA, the PA output varies by $0.26 \mathrm{~dB}$.
}

$3 \psi(t)]$, where $k$ denotes the relative strength of the third harmonics. The RF output is thus equal to

$$
\begin{aligned}
x_{\mathrm{TX}}(t)=a(t) \cos \left[\omega_{c} t+\phi(t)\right] \\
+k A_{\max } \cos \left[\omega_{c} t+3 \phi(t)\right] \cos [3 \psi(t)] .
\end{aligned}
$$

The second term is centered at $\omega_{c}$ but contains distorted envelope and phase. It must therefore remain sufficiently small.

Outphasing does relax the linearity at the output of the upconversion mixers. Compression at this port does not distort the signal, allowing large output swings and easing the task of PA design.

\section{B. Power Amplifier}

Most mm-wave PAs are designed with a $50-\Omega$ input, placing a heavy burden on the upconverter preceding them. ${ }^{11}$ To quantify this issue, suppose a PA has a gain of $10 \mathrm{~dB}$ and an output power of $+10 \mathrm{dBm}$. With a $50-\Omega$ input, the circuit draws an input power of $1 \mathrm{~mW}$, much less than that drawn from the supply. [The drain efficiency, $\eta$, and the power-added efficiency (PAE) are thus nearly equal.] On the other hand, the corresponding input voltage swing $\left(632 \mathrm{mV}_{\mathrm{pp}}\right)$, must be provided by the upconverter; i.e., a mixer must be designed that delivers $632 \mathrm{mV}_{\mathrm{pp}}$ to a $50-\Omega$ load. To this end, we consider the idealized arrangement shown in Fig. 13, where the drain current of $M_{1}$ swings between 0 and $2 I_{s s}$ (albeit producing high distortion), $M_{2}$ and $M_{3}$ switch abruptly, and the loss of $L_{1}, L_{2}$, and the requisite balun are neglected. We therefore have

$$
\begin{aligned}
V_{\text {out }, p p} & =2 I_{s s} \frac{2}{\pi} R_{L} \\
& =\frac{4}{\pi} I_{s s} R_{L}
\end{aligned}
$$

obtaining a bias current of $I_{s s}=9.9 \mathrm{~mA}$. To handle this current, the switching transistors must be large enough $(15 \mu \mathrm{m} / 60$ $\mathrm{nm}$ each), loading the quadrature VCO heavily. Circuit simulations of a $60-\mathrm{GHz}$ quadrature upconverter in $65-\mathrm{nm}$ technology with sinusoidal LO waveforms indicate the need for a total bias current of $16 \mathrm{~mA}$. That is, the upconverter power consumption becomes a significant fraction of the overall power drained by the TX.

In this work, the PA is designed with a relatively high input impedance $(70.5-346 j \Omega)$, and the input reactance resonates with the upconverter's load inductance, resulting in a net load resistance $\left(R_{L}\right.$ in Fig. 13) of $500 \Omega$. The quadrature upconverter in each TX therefore draws a supply current of only $5.8 \mathrm{~mA}$.

In order to deliver an output power of about $+10 \mathrm{dBm}$. the PA must incorporate large output transistors, on the order of $110 \mu \mathrm{m}$ in width (with a drawn length of $60 \mathrm{~nm}$ ). The large input capacitance in turn requires a low load inductance for the upconverter or a power hungry predriver for the output stage. This issue is greatly alleviated if the output stage drives some of its own input capacitance [26], a feasible concept if the signal path is differential. Depicted in Fig. 14, the one-stage PA consists of input devices $M_{3}-M_{4}$, and cross-coupled transistors,

\footnotetext{
${ }^{11}$ A passive matching network can be inserted between the PA and the mixer to optimize the load seen by the latter, but the loss of the network becomes problematic.
} 

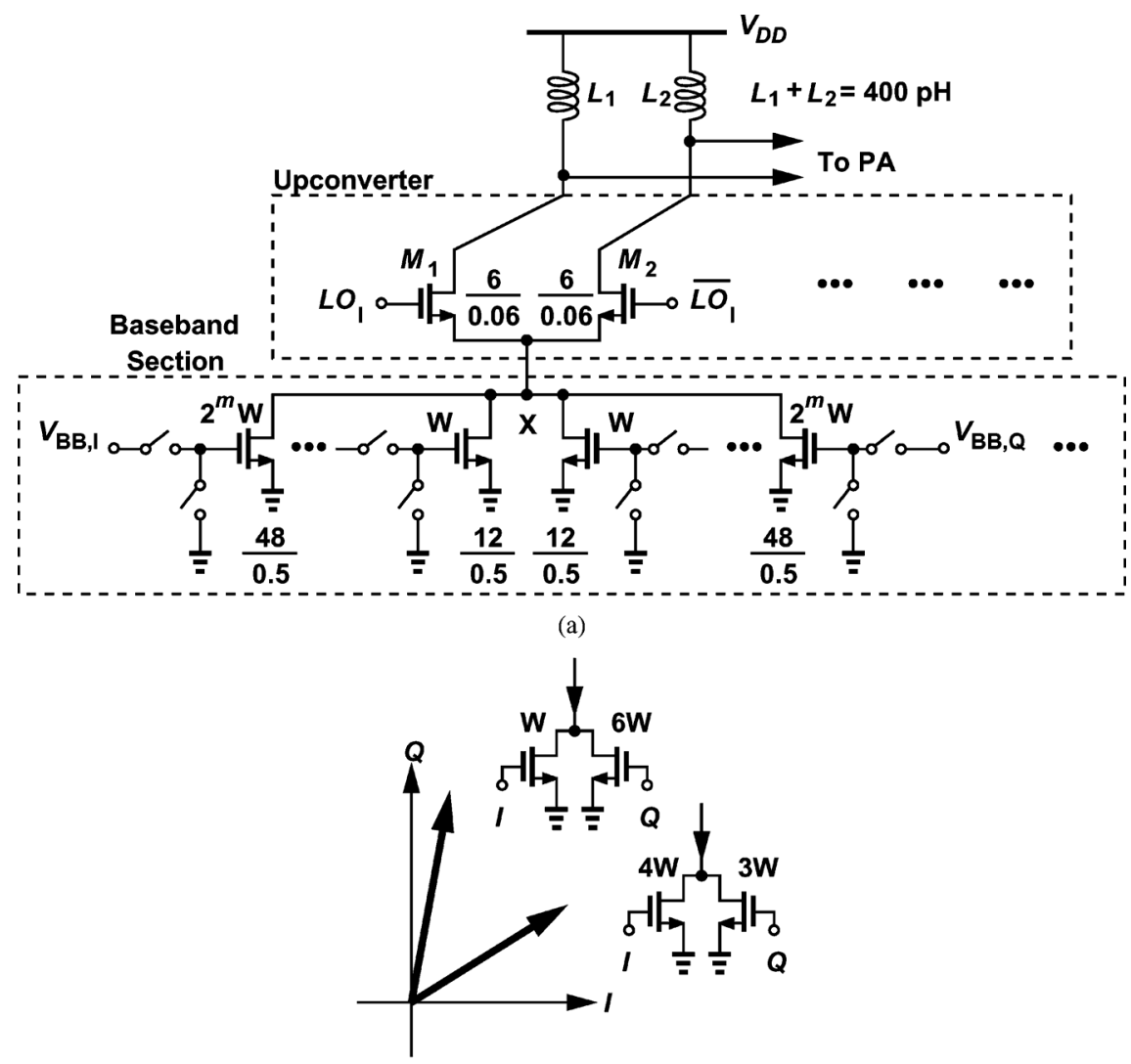

(b)

Fig. 12. (a) Proposed mixer with BB phase shifters and (b) configuration of the baseband devices for different interpolation codes.

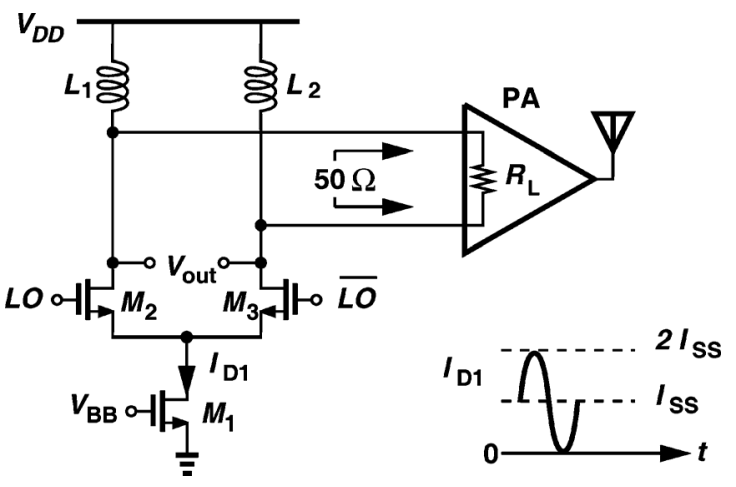

Fig. 13. Interface between upconverter and PA.

$M_{1}-M_{2}$. Here, $R_{P}$ models the loss of $L_{1}$ and $L_{2} ;$ and $R_{1}$ and $R_{2}\left(=3.5 \mathrm{k} \Omega\right.$ ) provide bias for the gates of $M_{1}$ and $M_{2}$. To simplify testing with external antennas, the output is applied to an on-chip balun consisting of a stack of single-turn metal 9 and metal 8 spirals. Unfortunately, the loss of the balun $(\approx 0.8 \mathrm{~dB})$ reduces the output power (and limits the maximum possible efficiency to $83 \%$ ). Note that $\mathrm{PAE} \approx \eta$ here.

The width of $M_{1}$ and $M_{2}$ in Fig. 14 is chosen according to the following observations. As $W_{1,2}$ increases (and $L_{1}=L_{2}=L$ decreases), the circuit can deliver a higher current to the load, thereby providing a greater output power. The efficiency also increases initially. However, if $W_{1,2}$ becomes so large that the requisite load inductance value translates to an $R_{P}$ not much greater than $R_{L} / 2$, then the output power reaches diminishing returns and the efficiency falls. Fig. 15 illustrates these trends based on transistor-level simulations of the circuit as $W_{1,2}$ goes from 30 to $220 \mu \mathrm{m}$ while $W_{3,4}=15 \mu \mathrm{m}$ and the $\mathrm{Q}$ of $L_{1}$ and $L_{2}$ is kept at 10 at $60 \mathrm{GHz} .^{12}$

The self-driving action of the output stage leads to two effects that must be handled carefully in the design. First, with sufficiently wide cross-coupled transistors, the circuit oscillates, operating as an injection-locked oscillator [26]. As indicated by Fig. 15, self-oscillation is inevitable if maximum $P_{\text {out }}$ and $\eta$ are sought. Thus, the lock range of the circuit must accommodate the input signal bandwidth and preferably the entire band of the communication channels. The simulated lock range of the PA is from 56 to $63 \mathrm{GHz}$, sufficient for typical signal bandwidths but not for the band of 57 to $64 \mathrm{GHz}$. Thus, some means of frequency tuning is necessary if the design must operate across the entire unlicensed band. As shown in Fig. 16(a), transistor-level simulations indicate that the PA can accommodate data rate as high as 3 Gbaud for EVM $=-20 \mathrm{~dB}$. Fig. 16(b) shows the simulated eye diagram after downconversion with a data rate of 2.5 Gbaud.

Second, with large transistors driven by the output nodes, the load inductors must be small, about $32 \mathrm{pH}$ in this work. This limitation manifests itself in the design of the balun: while, in principle, $L_{1}$ and $L_{2}$ in Fig. 14 can be omitted and the supply provided through the balun, the small primary inductance required

\footnotetext{
${ }^{12}$ This simulation does not include some of the distributed parasitics within the transistors, overestimating $P_{\text {out }}$ and $\eta$.
} 


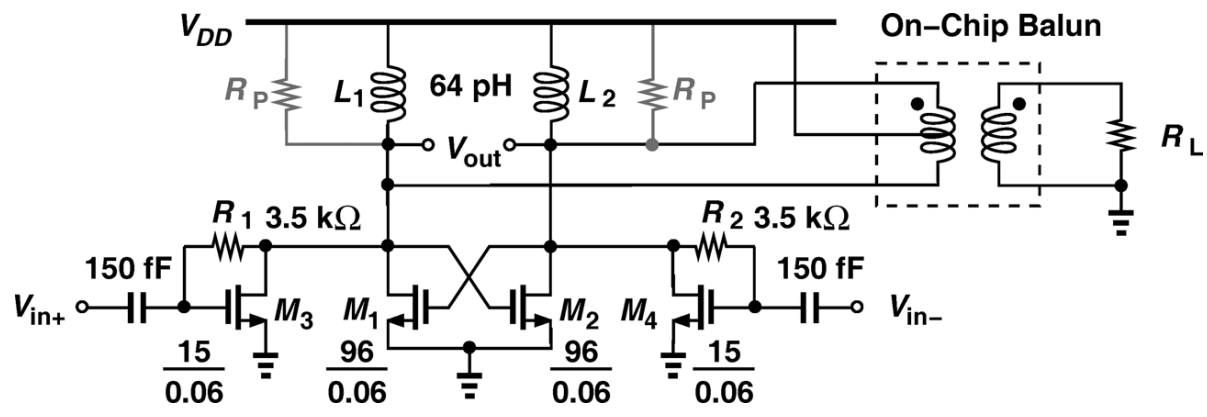

Fig. 14. PA circuit diagram.

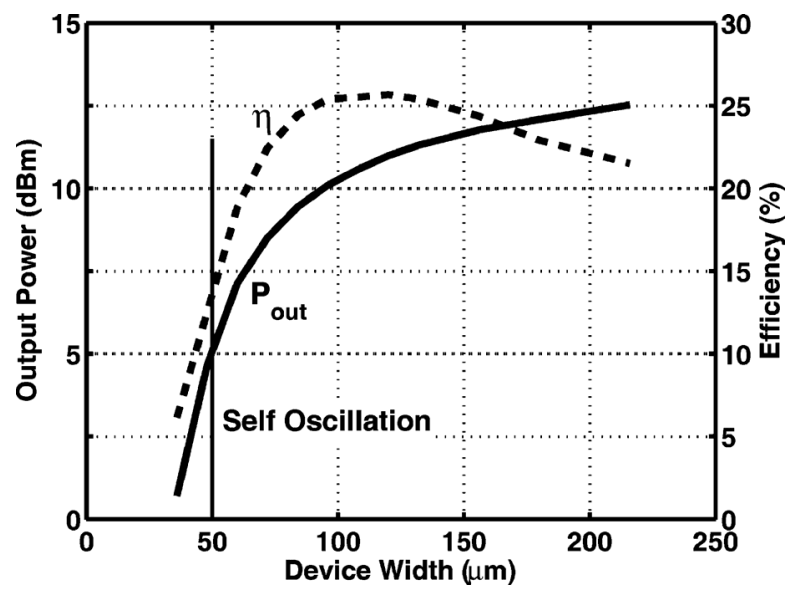

Fig. 15. Simulated PA performance with different transistor widths.

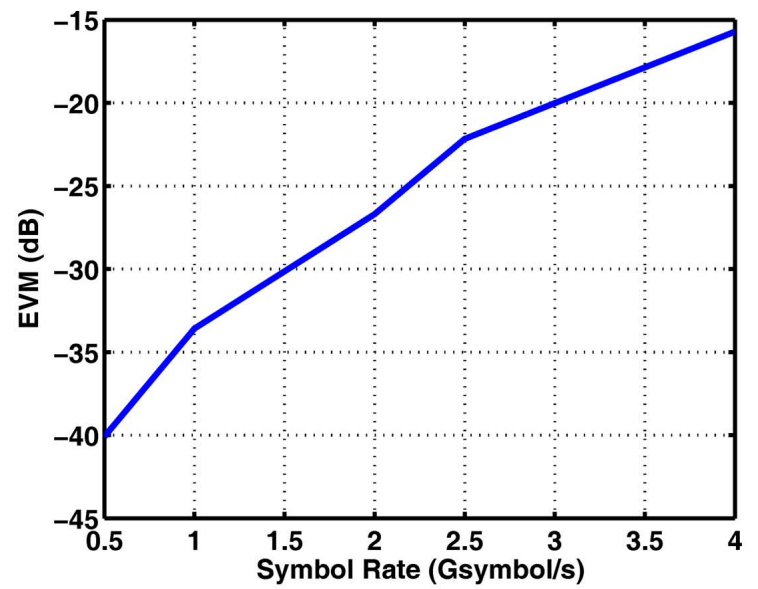

(a)

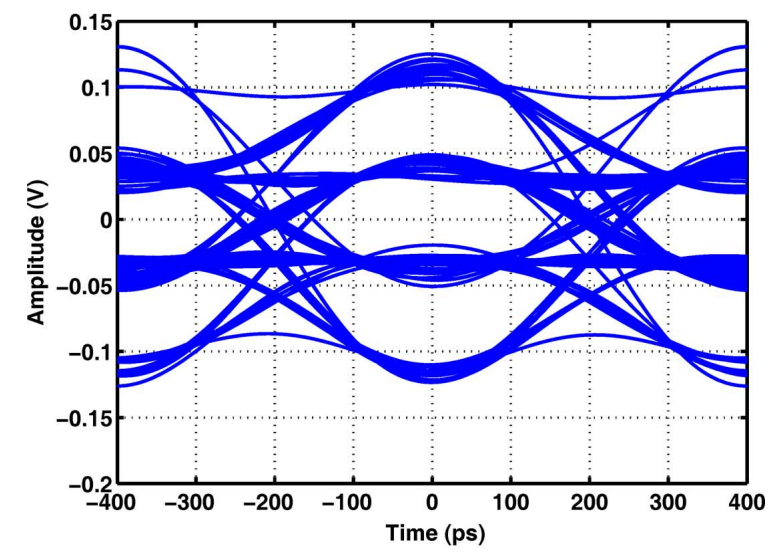

(b)

Fig. 16. (a) Simulated PA output EVM as a function of symbol rate (16QAM), demonstrating PA bandwidth and (b) simulated eye diagram after downconversion with a data rate of $2.5 \mathrm{Gsymbol} / \mathrm{s}$.

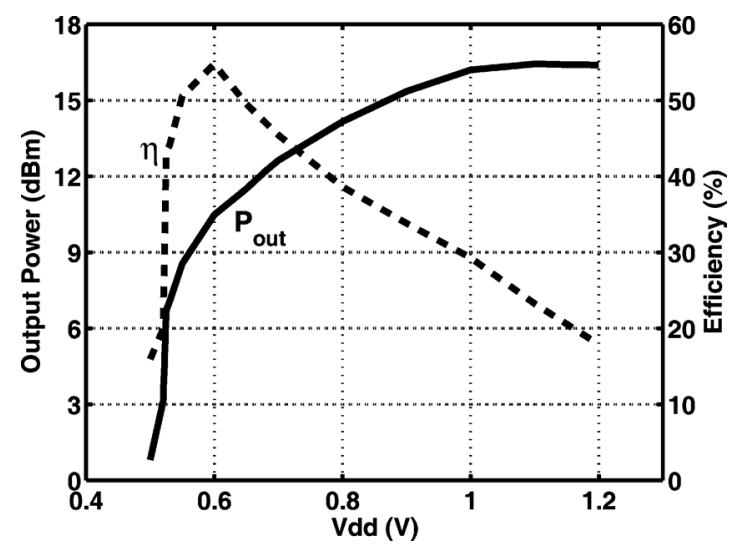

Fig. 17. Simulated PA performance with different supply voltages and with ideal passive devices.

of the balun would translate to a high loss. ${ }^{13}$ For the reason, the load inductors are retained and implemented with microstrips. The small de resistance of $L_{1}$ and $L_{2}$ also minimizes the de drop created by the large bias current of the output stage $(85 \mathrm{~mA})$, improving the efficiency. ${ }^{14}$

The PA stage of Fig. 14 senses a differential input voltage of $400 \mathrm{mV}_{\mathrm{pp}}$ and delivers approximately $+10 \mathrm{dBm}$ to the antenna. The self-driven, injection-locked operation can potentially yield a high efficiency. However, the simulated efficiency is about $25 \%$. It is therefore instructive to identify the causes of this efficiency degradation. We make the following observations. 1) With ideal, lossless inductors and balun, the efficiency is around $45.4 \%$. This is because the peak swing at the drain of $M_{1}$ and $M_{2}$ is equal to $560 \mathrm{mV}$ while $V_{\mathrm{DD}} \approx 0.7 \mathrm{~V}$, resulting in a minimum $V_{\mathrm{DS}}$ of $140 \mathrm{mV}$ when each transistor is on and hence a significant loss of power. If $V_{\mathrm{DD}}$ is lowered so that $V_{\mathrm{DS}}$ of a fully-conducting transistor comes closer to zero, the average output power falls. Fig. 17 plots the simulated $P_{\text {out }}$ and $\eta$ as a function of the output stage supply voltage in this idealized case. 2) With actual inductors and balun, a dc drop of $63.3 \mathrm{mV}$ appears from $V_{\mathrm{DD}}$ to each drain, $R_{P}=121 \Omega$, and the simulated balun has a loss of $0.83 \mathrm{~dB}$. Thus $\eta$ falls to $25 \%$. In the actual prototype, the layout parasitics, especially the gate resistance, the interconnect inductances, and resistances in series with the sources of the output devices further degrade the output power and efficiency. To restore a power level of $+10 \mathrm{dBm}$, the efficiency falls below $15 \%$.

${ }^{13}$ This is because, as the diameter of the spiral is reduced by a factor of $\beta$, the series resistance falls by the same factor whereas inductance drops by a factor of roughly $\beta^{2}$.

${ }^{14} \mathrm{HFSS}$ suggests a $\mathrm{Q}$ of 10.03 for $L_{1}$ and $L_{2}$, yielding $R_{p}=121 \Omega$. 


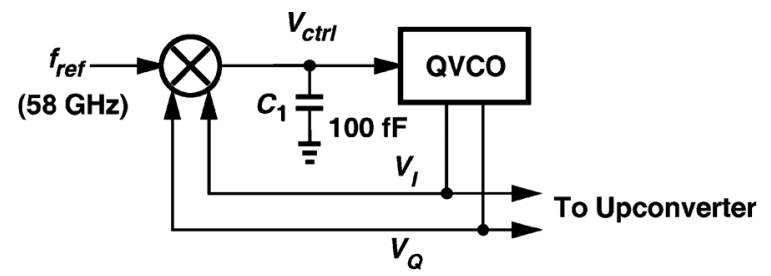

(a)

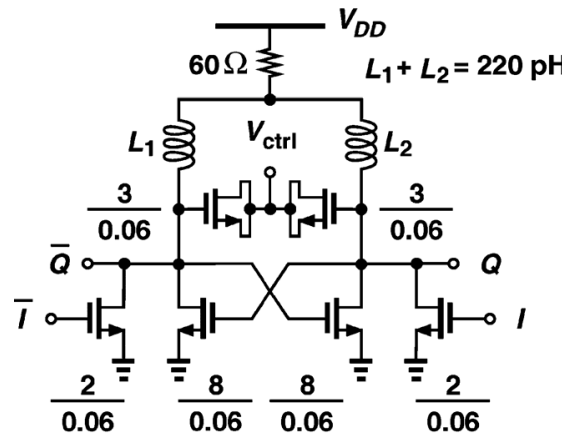

(b)

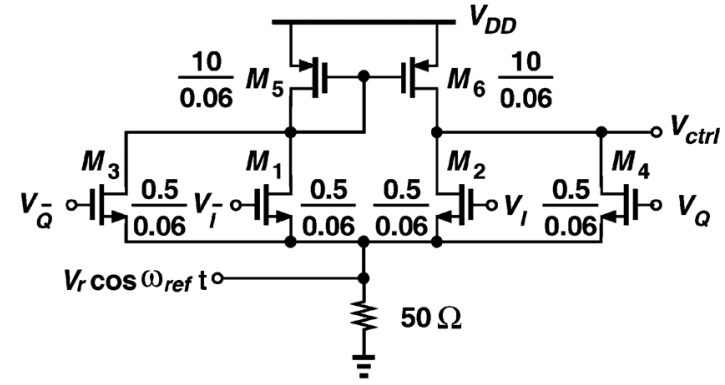

(c)

Fig. 18. (a) PLL topology, (b) one half of quadrature VCO, and (c) phase detector implementation.

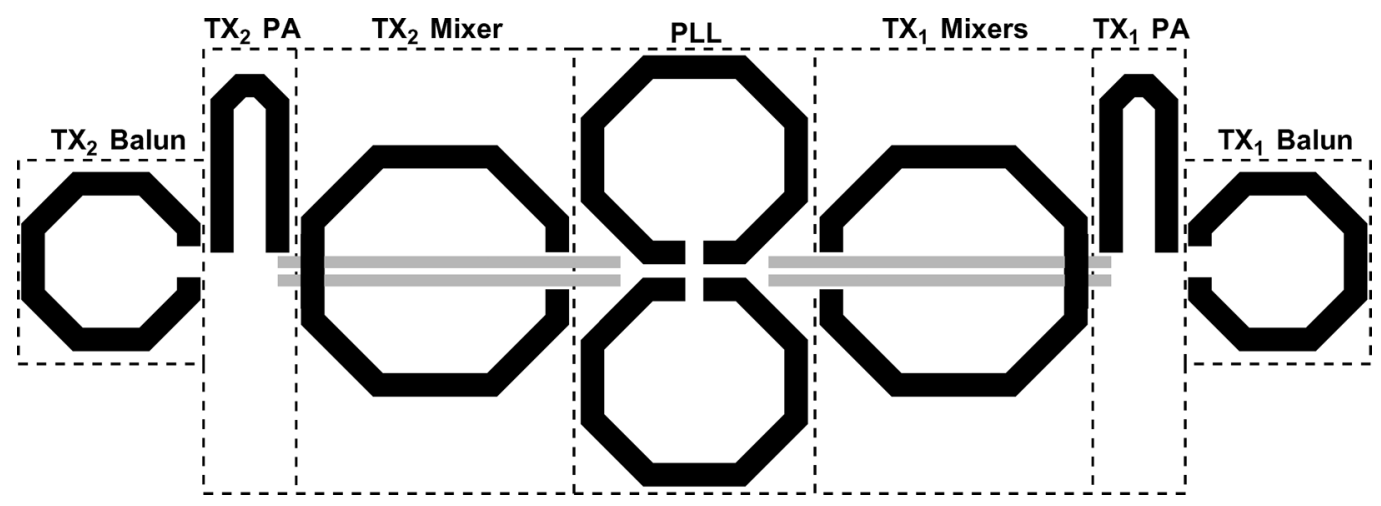

Fig. 19. Floor plan of prototype.

The use of large transistors in the PA also exacerbates the problem of layout parasitics. The metalization capacitances within each transistor can be lumped, added between each two terminals, and taken into account in the design [27]. On the other hand, the distributed inductances and resistances within the transistors can drastically degrade the performance due to the large currents flowing in the PA. Thus, each transistor is modeled as a multi-port network in Ansoft HFSS and returned to Cadence.

\section{PLL}

For testing flexibility, it is desirable to provide the $\mathrm{LO}$ waveforms externally. However, the need for accurate quadrature, differential LO phases dictates an on-chip oscillator. Furthermore, a free running oscillator would continue to drift with time, making signal constellation measurements difficult. For these reasons, an on-chip PLL drives both transmitters.

Fig. 18(a) shows the PLL diagram. To avoid risks associated with divider design, the PLL directly locks a quadrature VCO to an external (single-ended) input. Such a topology, however, requires a full-speed phase detector (PD) and lends itself to only a type-I PLL implementation. The PD circuit is

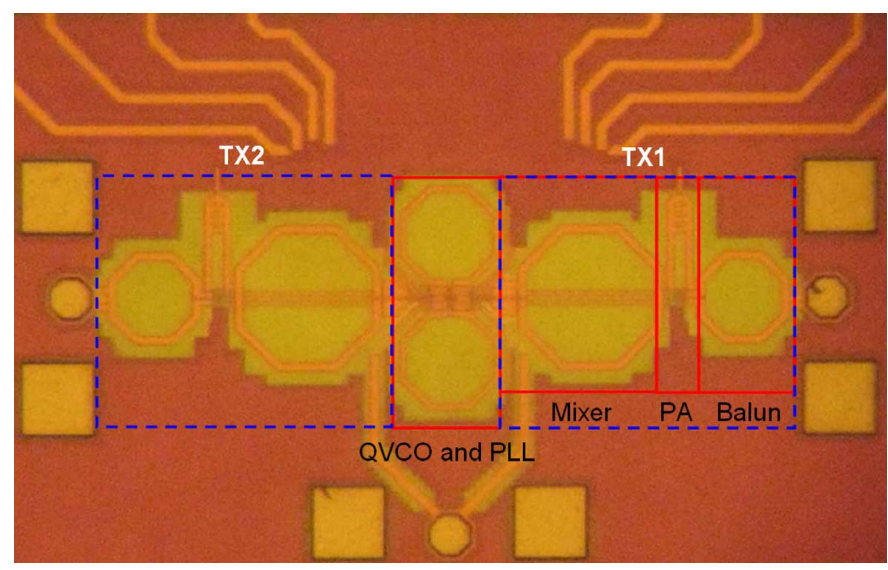

Fig. 20. Die photo of prototype.

depicted in Fig. 18(c). Here, each NMOS transistor mixes the input reference signal with one phase of the LO, loading the LO phases uniformly and generating a current proportional to the phase difference between the LO and the input. These currents are summed with proper polarities at the output node and flow through the output impedance of the circuit. The operation can be better seen by assuming nonlinear devices, $I_{D}=$ 

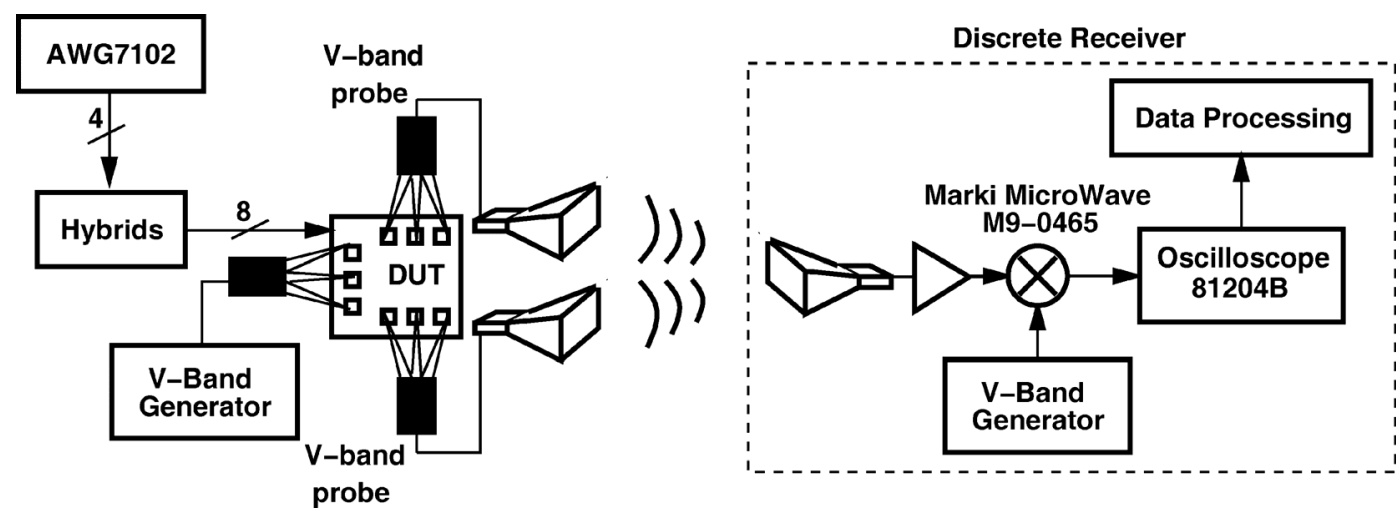

Fig. 21. Test setup.

$\alpha_{1}\left(V_{\mathrm{GS}}-V_{\mathrm{TH}}\right)+\alpha_{2}\left(V_{\mathrm{GS}}-V_{\mathrm{TH}}\right)^{2}+\alpha_{3}\left(V_{\mathrm{GS}}-V_{\mathrm{TH}}\right)^{3}$, writing $V_{I}=V_{0} \cos \left(\omega_{\mathrm{LO}} t+\theta\right)$, and $V_{Q}=V_{0} \sin \left(\omega_{\mathrm{LO}} t+\theta\right)$, and noting that $V_{\mathrm{GS} 1}=\overline{V_{I}}-V_{r} \cos \omega_{\text {ref }} t$, etc. The total current flowing to the output node therefore contains the following low frequency component:

$$
I_{\text {ctrl }}=\alpha_{2} V_{0} V_{r}\left[\cos \left(\omega_{\mathrm{LO}} t-\omega_{\text {ref }} t+\theta\right)+\sin \left(\omega_{\mathrm{LO}} t-\omega_{\text {ref }} t+\theta\right)\right] .
$$

When the system is locked, $I_{\mathrm{ctrl}}$ is near zero, forcing $\omega_{\mathrm{LO}}=$ $\omega_{\text {ref }}$ and $\theta$ towards $-\pi / 4$ or $+3 \pi / 4 . .^{15}$

The quadrature LC VCO is based on a standard coupled topology with 220-pH differential load inductors and small MOS varactors $(\mathrm{W} / \mathrm{L}=3 \mu \mathrm{m} / 60 \mathrm{~nm})$ so that it can directly drive two quadrature upconverters (a total width of $24 \mu \mathrm{m}$ ) and the PD transistors $(\mathrm{W}=0.5 \mu \mathrm{m})$. The $\mathrm{VCO}$ draws a total bias current of $10.5 \mathrm{~mA}$ and has a tuning range of $0.6 \mathrm{GHz}$.

\section{EXPERIMENTAL RESULTS}

The dual-TX prototype has been fabricated in TSMC's $65-\mathrm{nm}$ digital CMOS technology. Fig. 19 shows the floor plan of the prototype. The quadrature VCO symmetrically drives the two transmitters' upconverters. The unconverted signals (the gray lines) must inevitably travel through the upconverter load inductor to reach the PA stage. The transistor parasitics, the interconnects, and the inductors are modeled in HFSS as described in [27].

Fig. 20 shows the die photo; the active area is about $700 \mu \mathrm{m} \times 400 \mu \mathrm{m}$. The quadrature VCO operates at $58 \mathrm{GHz}$, and the transmitters are characterized for this carrier frequency. The prototype consumes a total of $193 \mathrm{~mW}$ from a 1-V supply: $5.8 \mathrm{~mW}$ in each upconverter, $85 \mathrm{~mW}$ in each PA, and $11 \mathrm{~mW}$ in the PLL. The dc and baseband pads have been bonded to a printed-circuit board, and the high-frequency pads are accessed through V-band probes.

Three sets of measurements are performed. For output power and efficiency, the output of one transmitter is directly applied to a V-band power meter. For the other two, namely, received spectrum and constellation measurements, the setup shown in Fig. 21 is used. Here, an arbitrary waveform generator (AWG)

\footnotetext{
${ }^{15}$ The PLL selects $\theta$ such that the slope of the mixer characteristic yields negative feedback around the loop.
}

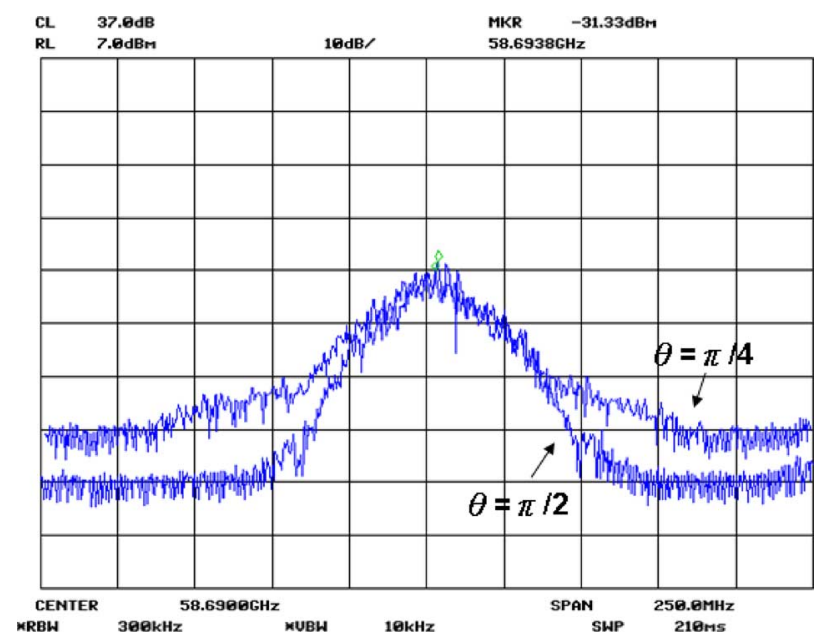

Fig. 22. Measured spectra received at $\theta=\pi / 2$ and $\pi / 4$ for a data rate of $200 \mathrm{Mb} / \mathrm{s}$.

produces the outphasing baseband signals and drives four hybrids, providing the eight components necessary for the two transmitters. (The AWG uses baseband pulse shaping with a raised-cosine filter and a roll-off factor of 0.5.) A V-band generator serves as the PLL reference. The TX outputs are applied to two horn antennas, and a third horn antenna receives the composite signal at different angles. For spectrum measurements, the receiving antenna simply drives a harmonic mixer and a spectrum analyzer. For constellation measurements, as shown in Fig. 21, the antenna drives a fundamental V-band mixer, generating an intermediate frequency of $312.5 \mathrm{MHz}$. This signal is digitized by an oscilloscope and taken to Matlab for processing. ${ }^{16}$ The maximum data rate is limited to $200 \mathrm{Mb} / \mathrm{s}$ by the bandwidth of the input hybrids.

Fig. 22 presents the measured spectra at $\theta=\pi / 2$ and $\pi / 4,{ }^{17}$ demonstrating that signal reconstruction yields a compact spectrum at the correct angle and a broad spectrum at other angles. ${ }^{18}$

\footnotetext{
${ }^{16}$ No off-line calibration is used except for correcting the phase offset between the on-chip LO and the external generators.

${ }^{17}$ Spectra at $\theta=\pi / 4$ and $\pi / 2$ are normalized for ease of comparison.

${ }^{18} \mathrm{At}$ angles away from the correct reception angle, the two outphasing signals combine incorrectly, violating the bandwidth savings afforded by 16QAM.
} 


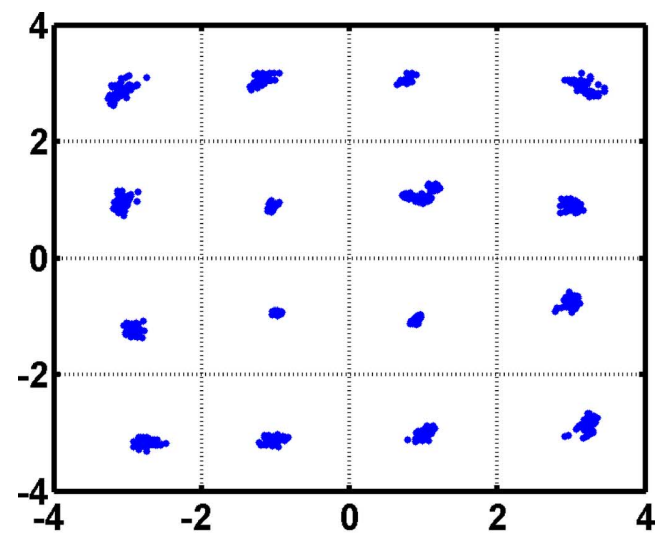

(a)

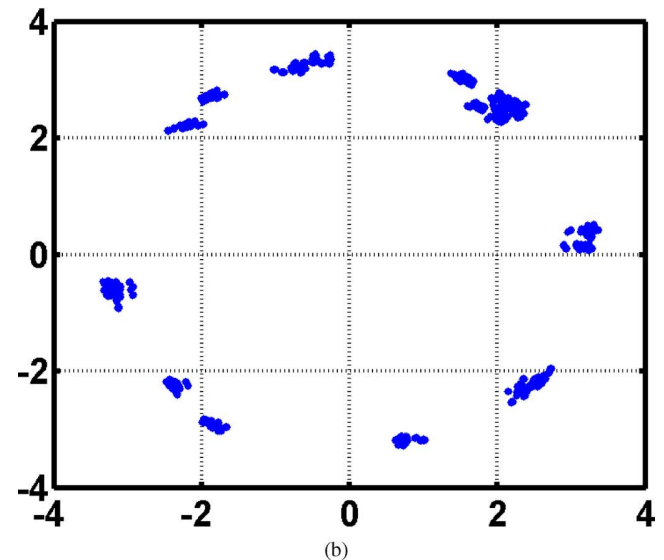

Fig. 23. Measured 16 QAM constellation of the data combination at (a) $\theta=$ $\pi / 2$ and (b) $2 \pi / 3$ for a data rate of $32 \mathrm{Mb} / \mathrm{s}$.
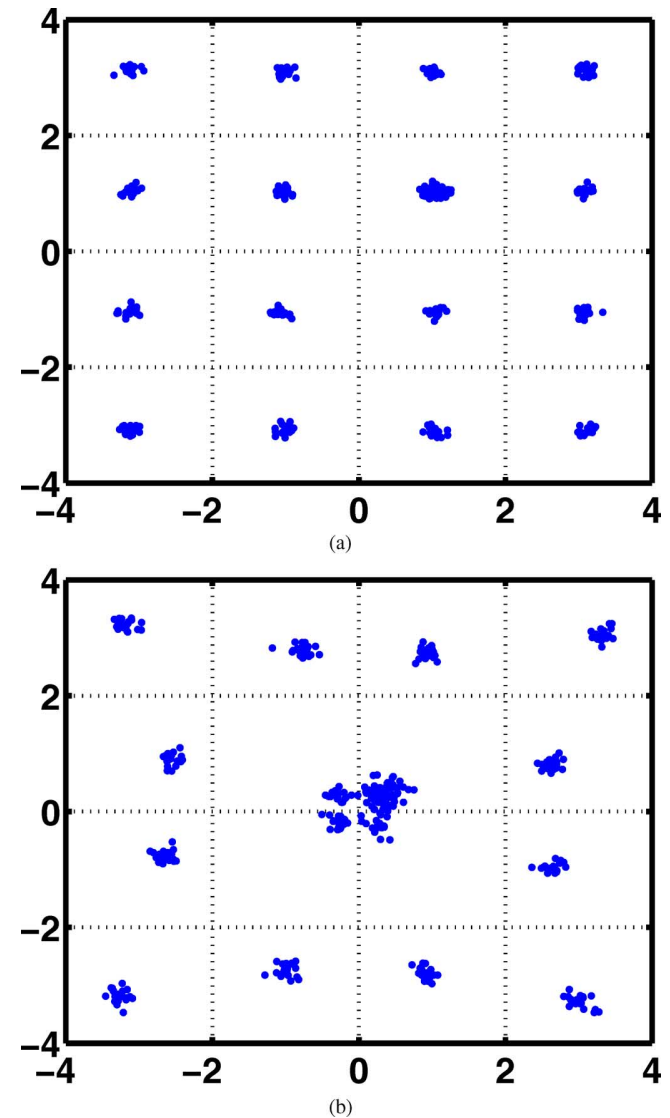

Fig. 24. Measured 16QAM constellation with (a) proper phase adjustment, (b) $10^{\circ}$ phase difference for a data rate of $200 \mathrm{Mb} / \mathrm{s}$.

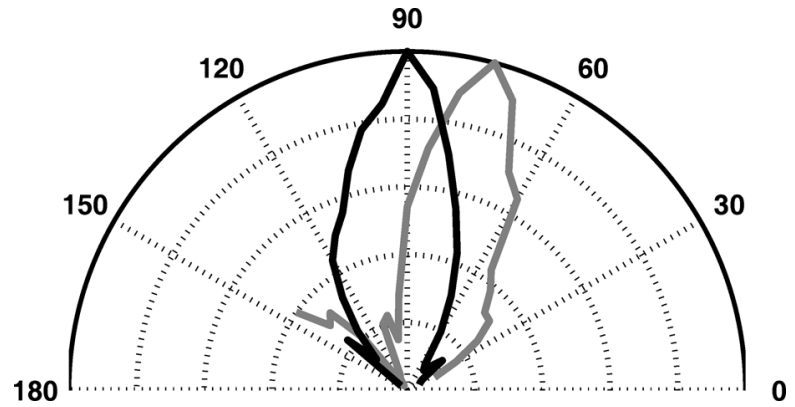

Fig. 25. Measured radiation patterns with baseband interpolation codes set to 0000 (black line) and 0011 (gray line).

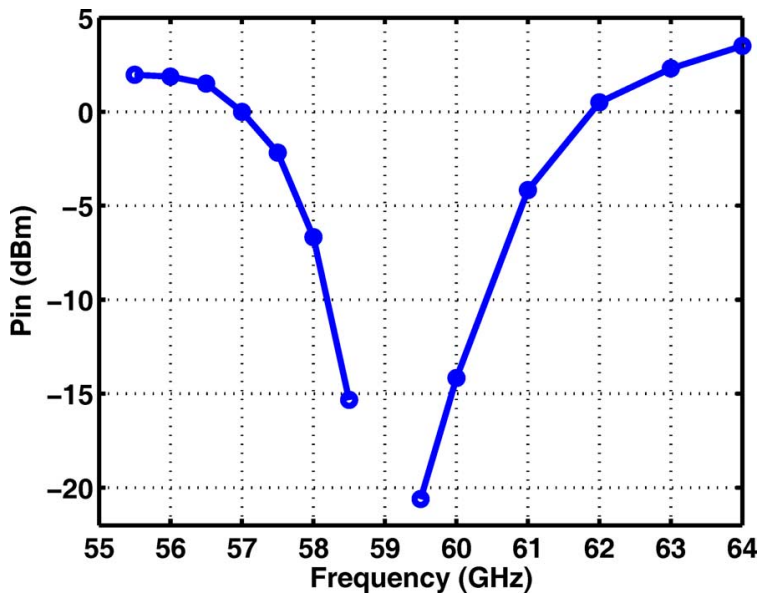

Fig. 26. Measured minimum required input power for lock as a function of frequency for the stand-alone PA.

Fig. 23 shows the measured received 16QAM constellation for a data rate of $32 \mathrm{Mb} / \mathrm{s}$. The EVM is equal to $-18.8 \mathrm{~dB}$. Here, the discrete receiver IF output is captured and digitized. The quality of the constellation in Fig. 23(a) also suggests that injection-pulling of the VCO by the PAs is negligible even with an output level of $+10 \mathrm{dBm}$ and with the poor isolation expected at $59 \mathrm{GHz}$.

Fig. 24 shows the measured 16QAM constellations with a data rate of $200 \mathrm{Mb} / \mathrm{s}$. The EVM is equal to $-21.6 \mathrm{~dB}$. Since it is difficult to align the phase of the received signals at this data rate (partially due to the finite resolution of the baseband phase shifters), the output of each transmitter is captured separately and phase adjustment and combining are performed in Matlab.

To verify the operation of the baseband phase shifters, the radiation pattern is measured with different interpolation codes. Fig. 25 shows the measured radiation patterns for codes 0000 and 0011. (In this case, two loop antennas with a spacing of $3 \lambda / 4$ have been fabricated on a printed-circuit board and bonded to the chip.)

To demonstrate the ability of the transmitter and, particularly, the PA to operate at high data rates, two measurements have been carried out. First, the injection lock range of the standalone PA is quantified by applying the minimum input level necessary for lock at different frequencies. (The PA prototype includes on-chip baluns at the input and the output.) Fig. 26 plots the measured results, suggesting, for example, a lock range of about $3.5 \mathrm{GHz}$ for an input level of $-4 \mathrm{dBm}$ (equivalent to 


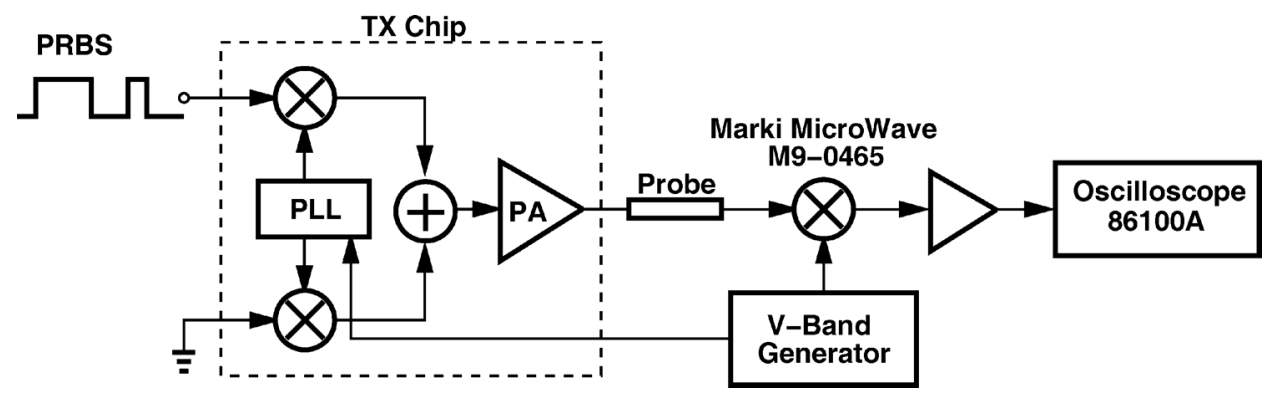

Fig. 27. Test setup to measure TX ability to operate with high data rates.

TABLE I

PA Performance Summary and COMPARISON AT $60 \mathrm{GHz}$-BAND

\begin{tabular}{|l|c|c|c|c|c|c|}
\hline Reference & {$[2]$} & {$[3]$} & {$[4]$} & {$[8]$} & This work & This work \\
\hline Technology & $45 \mathrm{~nm}$ & $90 \mathrm{~nm}$ & $90 \mathrm{~nm}$ & $65 \mathrm{~nm}$ & $65 \mathrm{~nm}$ & $65 \mathrm{~nm}$ \\
\hline P1dB & $11 \mathrm{dBm}$ & $10.2 \mathrm{dBm}$ & $9 \mathrm{dBm}$ & $2.5 \mathrm{dBm}$ & -- & -- \\
\hline Psat & $13.8 \mathrm{dBm}$ & $12.5 \mathrm{dBm}$ & $12.3 \mathrm{dBm}$ & $11.5 \mathrm{dBm}$ & $9.7 \mathrm{dBm}$ & $11.1 \mathrm{dBm}$ \\
\hline Max PAE & $7 \%$ & $19.3 \%$ & $8.8 \%$ & $11 \%$ & $11.0 \%$ & $8.9 \%$ \\
\hline Supply & $1.1 \mathrm{~V}$ & $1.2 \mathrm{~V}$ & $1 \mathrm{~V}$ & $1 \mathrm{~V}$ & $1 \mathrm{~V}$ & $1.2 \mathrm{~V}$ \\
\hline
\end{tabular}

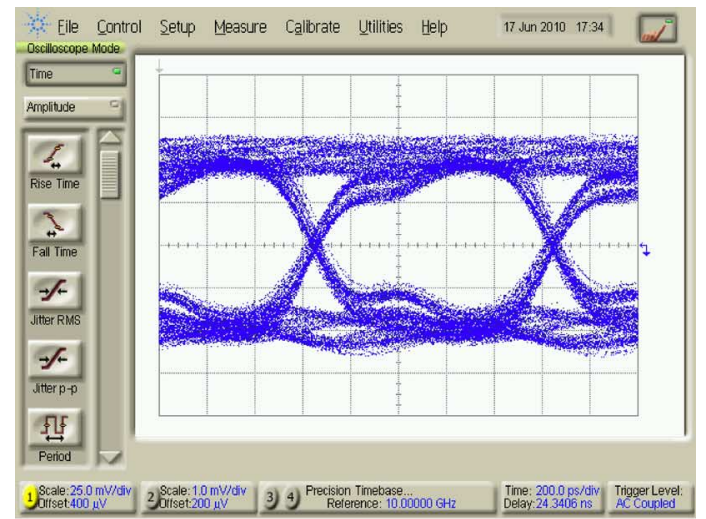

(a)

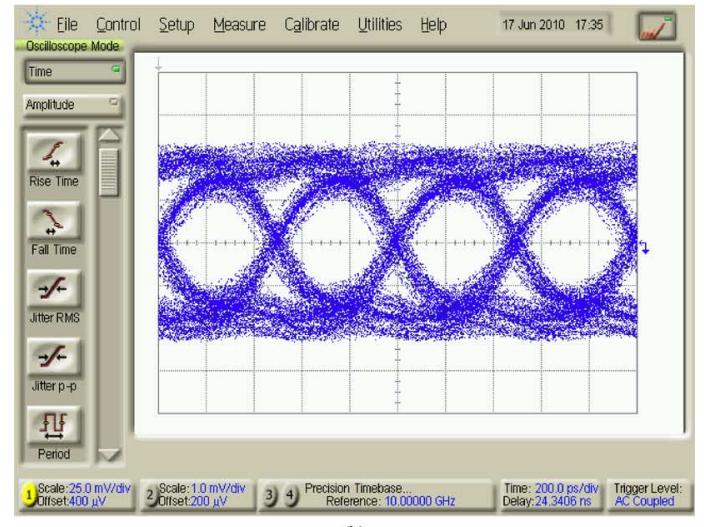

(b)

Fig. 28. Measured eye diagram for PRBS data after demodulation with a data rate of (a) $1 \mathrm{~Gb} / \mathrm{s}$ and (b) $2 \mathrm{~Gb} / \mathrm{s}$.

$400 \mathrm{mV}_{\mathrm{pp}}$, i.e., the output swing of the quadrature upconverter in the TX chain).

In the second test (Fig. 27), PRBS data at 1 or $2 \mathrm{~Gb} / \mathrm{s}$ is applied to one of the baseband mixers, creating a $60-\mathrm{GHz}$ BPSK signal after upconversion. This signal drives the PA (on the same chip) and the result is sensed at the output by a probe, which carries the modulated signal to an external mixer driven by a $58-\mathrm{GHz}$ LO. The baseband PRBS data produced by this mixer is then observed on an oscilloscope. Fig. 28 shows the measured eye diagrams in this test, indicating that the injection-locked PA easily accommodates phase-modulated signals at $2 \mathrm{~Gb} / \mathrm{s}$. (The data bandwidth is limited by the baseband input ESD structures.)

Table I summarizes the performance of our PA and that of prior art. Since the focus of this work is not PA design per se, our PA is not competitive with prior art at saturation. (As explained in Appendix I, outphasing relaxes the receiver noise figure by 2 dB.)

\section{CONCLUSION}

The use of outphasing at mm-wave frequencies can lead to power- and bandwidth-efficient communications. This paper has proposed a blend of outphasing and beamforming that avoids the loss of on-chip transformers and provides secure communications in a certain spatial angle. A self-driven injection-locked PA simplifies the design of the upconverter mixer and the PLL.

\section{APPENDIX I \\ BACK-OFF VERSUS BER}

The back-off necessary in a PA is determined by several factors: 1) the peak-to-average ratio of the variable-envelope signal, which itself depends on both the type of modulation and the baseband pulse shaping used to tighten the spectrum; 2) the bit error rate (a lower BER requires that the peaks of the waveform approach or exceed $P_{1 \mathrm{~dB}}$ less frequently); 3) the receiver signal-to-noise ratio (a higher SNR allows less back-off in the transmitter). A rigorous formulation must therefore reflect the trade-off between the TX back-off and the RX SNR. 


$$
\begin{aligned}
x_{\mathrm{RX}}(\tau) & =\sum_{i=1}^{n} \alpha_{i} A_{\max } \cos \left[\psi(\tau)+\omega_{c} \frac{d_{0} \cos \theta_{i}}{2 v_{c}}\right] \cos \left[\omega_{c} \tau+\phi(\tau)\right] \\
& =\sum_{i=1}^{n} \alpha_{i}\left[\cos \psi(\tau) \cos \left(\omega_{c} \frac{d_{0} \cos \theta_{i}}{2 v_{c}}\right)-\sin \psi(\tau) \sin \left(\omega_{c} \frac{d_{0} \cos \theta_{i}}{2 v_{c}}\right)\right] A_{\max } \cos \left[\omega_{c} \tau+\phi(\tau)\right] \\
& =\left[\cos \psi(\tau) \sum_{i=1}^{n} \alpha_{i} \cos \left(\omega_{c} \frac{d_{0} \cos \theta_{i}}{2 v_{c}}\right)-\sin \psi(\tau) \sum_{i=1}^{n} \alpha_{i} \sin \left(\omega_{c} \frac{d_{0} \cos \theta_{i}}{2 v_{c}}\right)\right] A_{\max } \cos \left[\omega_{c} \tau+\phi(\tau)\right] \\
& =\alpha \cos [\psi(\tau)+\epsilon] A_{\max } \cos \left[\omega_{c} \tau+\phi(\tau)\right]
\end{aligned}
$$

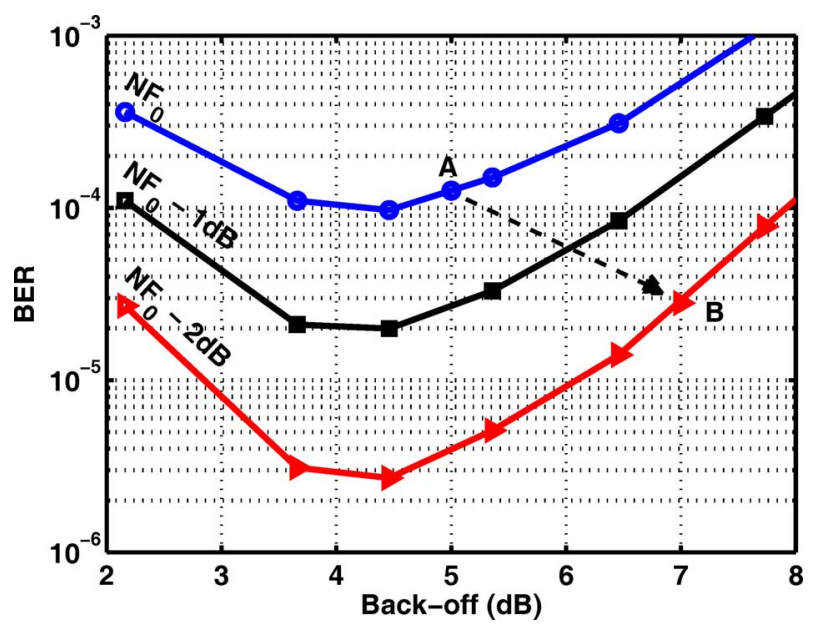

Fig. 29. BER for 16QAM signals with a roll-off factor of 0.4 in a nonlinear PA with back-off from $P_{1 \mathrm{~dB}}$.

To this end, we have employed the nonlinear PA model proposed by Rapp [28]:

$$
V_{\text {out }}=\frac{V_{\text {in }}}{\left(1+\left|\frac{V_{\text {in }}}{V_{\text {sat }}}\right|^{m}\right)^{1 / m}}
$$

where $m$ is an empirical value for accurate fitting. The inputoutput characteristics available in [2]-[4] suggest a value of approximately 3.5 for $m$. The simulation setup consists of 1) a PA with an input 16QAM signal whose average amplitude can be adjusted (to create different back-offs), and 2) a receiver whose noise figure can be adjusted. For each power level applied to the PA, the BER at the RX output is measured. Fig. 29 plots the results for a reference noise figure, $\mathrm{NF}_{0}$, and two lower values, $\mathrm{NF}_{0}-1 \mathrm{~dB}$ and $\mathrm{NF}_{0}-2 \mathrm{~dB} .{ }^{19}$ As expected, raising the input swing of the PA tends to improve the link performance up to a back-off of about $4.5 \mathrm{~dB}$ from $P_{1 \mathrm{~dB}}$, beyond which the distortion is too high to be corrected by the higher SNR.

It is important to note that the distortion penalty manifests itself even for a back-off of greater than $4.5 \mathrm{~dB}$. For example, points A and B in Fig. 29 correspond to equal RX SNRs (they differ by $2 \mathrm{~dB}$ in TX power and $2 \mathrm{~dB}$ in RX NF) but exhibit quite different BERs. In fact, simulations indicate that, in the

\footnotetext{
${ }^{19}$ The path loss and $\mathrm{NF}_{0}$ are chosen such that the RX SNR is equal to $16.6 \mathrm{~dB}$ at 7.5-dB back-off from $P_{1 \mathrm{~dB}}\left(\mathrm{BER}=10^{-3}\right)$.
}

absence of nonlinearity, a BER of $10^{-4}$ can be obtained with an $\mathrm{RX}$ noise figure equal to $\mathrm{NF}_{0}+2 \mathrm{~dB}$.

The foregoing observation reveals the advantage of outphasing over a traditional PA with back-off: for a given RX noise figure and BER, the signal received from an outphasing system can be $2 \mathrm{~dB}$ lower than that from a traditional PA operating at $4.5-\mathrm{dB}$ back-off. ${ }^{20}$ The noise figure advantage depends on factors such as the type of modulation and the nonlinearity of the PA. The value of $2 \mathrm{~dB}$ obtained here is for 16QAM and the PA model given by (21).

\section{APPENDIX II MulTipATH EFFECTS}

Conventional beamforming system can employ orthogonal frequency division multiplexing (OFDM) in each transmitter to deal with multipath effects. For a conventional beamforming system, multipath effects only attenuate the amplitude if OFDM is used. In our system, on the other hand, since signals are only correctly combined in certain directions, the signal reflected from an undesired direction may cause corruption. If OFDM is used, the received signals in a multipath environment can be written from (7) as shown in the equation at the top of the page, where

$$
\begin{aligned}
& \alpha=\sum_{i=1}^{n} \alpha_{i}^{2}+2 \sum_{\substack{i=1, j=1 \\
i \neq j}}^{n} \alpha_{i} \alpha_{j} \cos \left(\omega_{c} \frac{d_{0} \cos \theta_{i}}{2 v_{c}}-\omega_{c} \frac{d_{0} \cos \theta_{j}}{2 v_{c}}\right) \\
& \epsilon=\tan ^{-1} \frac{\sum_{i=1}^{n} \alpha_{i} \sin \left(\omega_{c} \frac{d_{0} \cos \theta_{i}}{2 v_{c}}\right)}{\sum_{i=1}^{n} \alpha_{i} \cos \left(\omega_{c} \frac{d_{0} \cos \theta_{i}}{2 v_{c}}\right)} .
\end{aligned}
$$

By adding a phase shift $\Delta \phi=-\epsilon$ in transmitters, signals received at $\theta$ can be correctly decoded. To obtain the phase shift caused by multipath, pilot signals can be sent before communication.

\section{ACKNOWLEDGMENT}

The authors gratefully appreciate the TSMC University Shuttle Program for fabrication support.

\section{REFERENCES}

[1] J. S. Seybold, "Output back-off requirements for root-raised cosinefiltered digital signals," RF Signal Processing, pp. 50-58, Jun. 2002.

${ }^{20}$ If distortion effects in outphasing (e.g., AM/PM conversion) are neglected. 
[2] K. Raczkowski, S. Thijs, W. D. Raedt, B. Nauwelaers, and P. Wambacq, "50-to-67 GHz ESD-protected power amplifiers in digital 45 nm LP CMOS," in 2009 IEEE ISSCC Dig. Tech. Papers, Feb. 2009, pp. 382-383.

[3] T. LaRocca and M. F. Chang, " $60 \mathrm{GHz}$ CMOS differential and transmormer-coupled power amplifier for compact design," in 2008 IEEE Radio Frequency Integrated Circuits (RFIC) Symp. Dig., Jun. 2008, pp. 65-68.

[4] D. Chowdhury, P. Reynaert, and A. Niknejad, "A $60 \mathrm{GHz} 1 \mathrm{~V}+12.3$ $\mathrm{dBm}$ transformer-coupled wideband PA in $90 \mathrm{~nm}$ CMOS," in 2008 IEEE ISSCC Dig. Tech. Papers, Feb. 2008, pp. 560-635.

[5] M. Tanomura, Y. Hamada, S. Kishimoto, M. Ito, N. Orihashi, K. Maruhashi, and H. Shimawaki, "Tx and Rx front-ends for $60 \mathrm{GHz}$ band in $90 \mathrm{~nm}$ standard bulk CMOS," in 2008 IEEE ISSCC Dig. Tech. Papers, Feb. 2008, pp. 558-635.

[6] S. Pinel, S. Sarkar, P. Sen, B. Perumana, D. Yeh, D. Dawn, and J. Laskar, "A $90 \mathrm{~nm}$ CMOS $60 \mathrm{GHz}$ radio," in 2008 IEEE ISSCC Dig. Tech. Papers, Feb. 2008, pp. 130-601.

[7] T. Suzuki et al., " 60 and $70 \mathrm{GHz}$ power amplifiers in standard 90 nm CMOS," in 2008 IEEE ISSCC Dig. Tech. Papers, Feb. 2008, pp. $562-563$.

[8] W. L. Chan, J. R. Long, M. Spirito, and J. J. Pekarik, "A 60 GHz-band $1 \mathrm{~V} 11.5 \mathrm{dBm}$ power amplifier with $11 \%$ PAE in $65 \mathrm{~nm}$ CMOS," in 2009 IEEE ISSCC Dig. Tech. Papers, Feb. 2009, pp. 380-381.

[9] L. R. Kahn, "Single-sideband transmission by envelope elimination and restoration," Proc. IRE, vol. 40, pp. 803-806, Jul. 1952.

[10] H. Chireix, "High power outphasing modulation," Proc. IRE, vol. 23, pp. 1370-1392, Nov. 1935

[11] D. C. Cox, "Linear amplification with nonlinear components," IEEE Trans. Commun., vol. COM-22, pp. 1942-1945, Dec. 1974.

[12] T. Sowlati, D. Rozenblit, R. Pullela, M. Damgaard, E. McCarthy, D. Koh, D. Ripley, F. Balteanu, and I. Gheorghe, "Quad-band GSM/GPRS/EDGE polar loop transmitter," IEEE J. Solid-State Circuits, vol. 39, no. 12, pp. 2179-2189, Dec. 2004.

[13] M. R. Elliott, T. Montalvo, B. P. Jeffries, F. Murden, J. Strange, A. Hill, S. Nandipaku, and J. Harrebek, "A polar modulator transmitter for GSM/EDGE," IEEE J. Solid-State Circuits, vol. 39, no. 12, pp. 2190-2199, Dec. 2004.

[14] S. Moloudi, K. Takinami, M. Youssef, M. Mikhemar, and A. Abidi, "An outphasing power amplifier for a software-defined radio transmitter," in 2008 IEEE ISSCC Dig. Tech. Papers, Feb. 2008, pp. $568-636$.

[15] A. Pham and C. G. Sodini, "A $5.8 \mathrm{GHz}, 47 \%$ efficiency, linear outphase power amplifier with fully integrated power combiner," in 2006 IEEE Radio Frequency Integrated Circuits (RFIC) Symp. Dig., Jun. 2006, pp. $157-160$.

[16] A. Babakhani, D. B. Rutledge, and A. Hajimiri, "Transmitter architectures based on near-field direct antenna modulation," IEEE J. SolidState Circuits, vol. 43, pp. 2674-2692, 2008.

[17] B. Razavi, RF Microelectonics. Englewood Cliffs, NJ: Prentice-Hall, 1998.

[18] J. Paramesh, R. Bishop, K. Soumyanath, and D. J. Allstot, "A fourantenna receiver in $90 \mathrm{~nm}$ CMOS for beamforming and spatial diversity," IEEE J. Solid-State Circuits, vol. 40, no. 12, pp. 2515-2524, Dec. 2005.

[19] A. Natarajan, A. Komijani, X. Guan, A. Babakhani, and A. Hajimiri, "A 77-GHz phased-array transceiver with on-chip antennas in silicon: Transmitter and local LO-path phase shifting," IEEE J. Solid-State Circuits, vol. 41, no. 12, pp. 2807-2819, Dec. 2006.

[20] A. Babakhani, X. Guan, A. Komijani, A. Natarajan, and A. Hajimiri, "A 77-GHz phased array transceiver with on-chip antennas in silicon: Receiver and antennas," IEEE J. Solid-State Circuits, vol. 41, no. 12, pp. 2795-2806, Dec. 2006.

[21] S. Kishimoto, N. Orihashi, Y. Hamada, M. Ito, and K. Maruhashig, "A $60-\mathrm{GHz}$ band CMOS phased array transmitter utilizing compact baseband phase shifters," in 2009 IEEE Radio Frequency Integrated Circuits (RFIC) Symp. Dig., Jun. 2009, pp. 215-218.

[22] K. Raczkowski, W. D. Raedt, B. Nauwelaers, and P. Wambacq, "A wideband beamformer for a phased-array $60 \mathrm{GHz}$ receiver in $40 \mathrm{~nm}$ digital CMOS," in 2010 IEEE ISSCC Dig. Tech. Papers, Feb. 2010, pp. $40-41$.

[23] T. Chu, J. Roderick, and H. Hashemi, "An integrated ultra-wideband timed array in $0.13 \mathrm{~mm}$ CMOS using a path sharing true time delay architecture," IEEE J. Solid-State Circuits, vol. 42, no. 12, pp. 2834-2850, Dec. 2007.
[24] C.-H. Lin, F. van der Goes, J. Westra, J. Mulder, Y. Lin, E. Arslan, E. Ayranci, X. Liu, and K. Bult, "A 12 b 2.9 GS/s DAC with $\operatorname{Im} 3<-60$ $\mathrm{dBc}$ beyond $1 \mathrm{GHz}$ in $65 \mathrm{~nm}$ CMOS," in 2009 IEEE ISSCC Dig. Tech. Papers, Feb. 2009, pp. 74-75.

[25] C. Marcu et al., "A $90 \mathrm{~nm}$ CMOS low-power $60 \mathrm{GHz}$ transceiver with integrated baseband circuitry," in 2009 IEEE ISSCC Dig. Tech. Papers, Feb. 2009, pp. 314-315.

[26] K. Tsai and P. R. Gray, "A 1.9-GHz, 1-W CMOS class-E power amplifier for wireless communications," IEEE J. Solid-State Circuits, vol. 34, no. 7, pp. 962-970, Jul. 1999.

[27] C. K. Liang and B. Razavi, "Systematic transistor and inductor modeling for millimter-wave design," IEEE J. Solid-State Circuits, vol. 44, no. 2, pp. 450-457, Feb. 2009.

[28] C. Rapp, "Effects of HPA-nonlinearity on a 4-DPSK/OFDM-signal for a digital sound broadband system," in Rec. Conf. ECSC'91, Liege, Belgium, Oct. 1991, pp. 179-184.

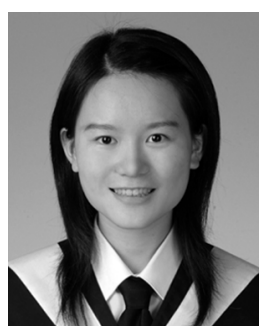

ChuanKang Liang (S'05-M'10) received the B.S. and M.S. degrees in electrical engineering from National Taiwan University (NTU), Taiwan, in 2004 and 2006, respectively, and the Ph.D. degree in electrical engineering from the University of California, Los Angeles, in 2009. Her Ph.D. research includes the architecture and system design of high-speed transceivers, RFICs for wireless communications, and device modeling for $\mathrm{mm}$-wave circuits. She is now with Mediatek USA, working on mm-wave transceivers.

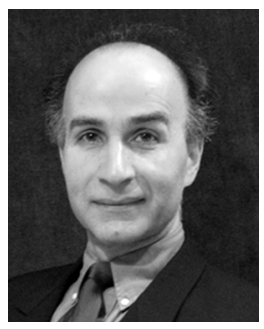

Behzad Razavi (M'90-SM'00-F'03) received the B.S.E.E. degree from Sharif University of Technology, Tehran, Iran, in 1985 and the M.S.E.E. and Ph.D.E.E. degrees from Stanford University, Stanford, CA, in 1988 and 1992, respectively.

He was with AT\&T Bell Laboratories and HewlettPackard Laboratories until 1996. Since 1996, he has been Associate Professor and subsequently Professor of electrical engineering at the University of California, Los Angeles. He was an Adjunct Professor at Princeton University from 1992 to 1994, and at Stanford University in 1995. His current research includes wireless transceivers, frequency synthesizers, phase-locking and clock recovery for high-speed data communications, and data converters.

Prof. Razavi served on the Technical Program Committees of the IEEE International Solid-State Circuits Conference (ISSCC) from 1993 to 2002 and the VLSI Circuits Symposium from 1998 to 2002. He has also served as Guest Editor and Associate Editor of the IEEE JOURNAL OF SOLID-STATE CIRCUITS, IEEE TRANSACTIONS ON CIRCUITS AND SYSTEMS, and the International Journal of High Speed Electronics. He received the Beatrice Winner Award for Editorial Excellence at the 1994 ISSCC, the Best Paper Award at the 1994 European Solid-State Circuits Conference, the Best Panel Award at the 1995 and 1997 ISSCC, the TRW Innovative Teaching Award in 1997, the Best Paper Award at the IEEE Custom Integrated Circuits Conference (CICC) in 1998, and the McGraw-Hill First Edition of the Year Award in 2001. He was the co-recipient of both the Jack Kilby Outstanding Student Paper Award and the Beatrice Winner Award for Editorial Excellence at the 2001 ISSCC. He received the Lockheed Martin Excellence in Teaching Award in 2006, the UCLA Faculty Senate Teaching Award in 2007, and the CICC Best Invited Paper Award in 2009 . He was also recognized as one of the top 10 authors in the 50 -year history of ISSCC.

Prof. Razavi is an IEEE Distinguished Lecturer, a Fellow of IEEE, and the author of Principles of Data Conversion System Design (IEEE Press, 1995), RF Microelectronics (Prentice Hall, 1998) (translated to Chinese, Japanese, and Korean), Design of Analog CMOS Integrated Circuits (McGraw-Hill, 2001) (translated to Chinese, Japanese, and Korean), Design of Integrated Circuits for Optical Communications (McGraw-Hill, 2003), and Fundamentals of Microelectronics (Wiley, 2006) (translated to Korean and Portuguese), and the editor of Monolithic Phase-Locked Loops and Clock Recovery Circuits (IEEE Press, 1996) and Phase-Locking in High-Performance Systems (IEEE Press, 2003). 